AcCePted for Publication in The Astrophysical Journal

Preprint typeset using $\mathrm{LAT}_{\mathrm{E}} \mathrm{X}$ style emulateapj v. 03/07/07

\title{
INTEGRAL FIELD SPECTROSCOPY OF BLUE COMPACT DWARF GALAXIES
}

\author{
Begoña García-Lorenzo \\ Instituto de Astrofísica de Canarias, E-38200 La Laguna, Tenerife, Canary Islands, Spain \\ Luz M. CAIRÓS \\ Astrophysikalisches Institut Potsdam, An der Sternwarte 16, D-14482 Potsdam, Germany \\ Nicola CAON \\ Instituto de Astrofísica de Canarias, E-38200 La Laguna, Tenerife, Canary Islands, Spain \\ Ana Monreal-Ibero \\ Instituto de Astrofísica de Canarias, E-38200 La Laguna, Tenerife, Canary Islands, Spain \\ Carolina Kehrig \\ Instituto de Astrofísica de Andalucía, C. Bajo de Huetor, E-18008 Granada, Spain \\ Accepted for publication in The Astrophysical Journal
}

\begin{abstract}
We present results on integral-field optical spectroscopy of five luminous Blue Compact Dwarf galaxies. The data were obtained using the fiber system INTEGRAL attached at the William Herschel telescope. The galaxies Mrk 370, Mrk 35, Mrk 297, Mrk 314 and III Zw 102 were observed. The central $33^{\prime \prime} .6 \times 29^{\prime \prime} .4$ regions of the galaxies were mapped with a spatial resolution of $2^{\prime \prime} .7 \mathrm{spaxel}^{-1}$, except for Mrk 314, in which we observed the central $16^{\prime \prime} \times 12^{\prime \prime}$ region with a resolution of $0^{\prime \prime} .9$ spaxel $^{-1}$. We use high-resolution optical images to isolate the star-forming knots in the objects; line ratios, electron densities and oxygen abundances in each of these regions are computed. We build continuum and emission-line intensity maps as well as maps of the most relevant line ratios: [O III $] \lambda 5007 / \mathrm{H} \beta$, [N II] $\lambda 6584 / \mathrm{H} \alpha$, and $\mathrm{H} \alpha / \mathrm{H} \beta$, which allow us to obtain spatial information on the ionization structure and mechanisms. We also derive the gas velocity field from the $\mathrm{H} \alpha$ and [O III] $\lambda 5007$ emission lines.

We find that all the five galaxies are in the high end of the metallicity range of Blue Compact Dwarf galaxies, with oxygen abundances varying from $Z_{\odot} \simeq 0.3$ to $Z_{\odot} \simeq 1.5$. The objects show $\mathrm{H}$ II-like ionization in the whole field of view, except the outer regions of III Zw 102 whose large [N II] $\lambda 6584 / \mathrm{H} \alpha$ values suggest the presence of shocks. The five galaxies display inhomogeneous extinction patterns, and three of them have high $\mathrm{H} \alpha / \mathrm{H} \beta$ ratios, indicative of a large dust content; all galaxies display complex, irregular velocity fields in their inner regions.
\end{abstract}

Subject headings: galaxies: dwarf - galaxies: evolution - galaxies: starburst - galaxies: kinematics

\section{INTRODUCTION}

Blue Compact Dwarf (BCD) galaxies are lowluminosity and compact objects, which show optical spectra similar to those presented by $\mathrm{H}$ II regions in spiral galaxies (Thuan \& Martin 1981). They are also low-metallicity systems (Searle \& Sargent 1972; Lequeux et al. 1979; Kunth \& Sargent 1983; Masegosa, Moles, \& Campos-Aguilar 1994) with intense star formation activity (rates ranging between 0.1 and $1 M_{\odot} \mathrm{yr}^{-1}$; Fanelli. O'Connell, \& Thuan 1988). In most BCDs the gas consumption time scale is much shorter than the age of the Universe, which, together with their low metal content, raised initially the hypothesis that they could be truly young galaxies, forming their first generation of stars (Searle \& Sargent 1972; Kunth. Maurogordato, \& Vigroux 1988). Nowa-

Electronic address: bgarcia@iac.es

Electronic address: luzma@aip.de

Electronic address: nicola.caon@iac.es

Electronic address: amonreal@iac.es

Electronic address: kehrig@iaa.es days it is clear that the great majority of them are not young, but rather old systems (Papaderos et al. 1996a; Cairós et al. 2001a, =C01a; Cairós et al. 2001b, =C01b; Bergvall \& Ostlin 2002; Cairós et al. 2003, =C03; Gil de Paz et al. 2003) undergoing short starbursts followed by longer quiescent periods.

An essential requisite to comprehend the formation and evolution of BCDs, and to elaborate coherent pictures of dwarf galaxy evolution, is to understand the process of star-formation in these galaxies - how their starburst activity ignites and how it propagates afterward - , and to derive their star-forming (SF) histories. Even though both issues have been the subject of a considerable observational and interpretative effort during the last years, they are still far from being well understood.

Different scenarios have been invoked to explain the onset of star formation in these galaxies. Some of them favored internal processes, such as the Stochastic Self-Propagating Star Formation proposed by Gerola, Seiden, \& Schulman (1980), or the hy- 
pothesis of cyclic gas re-processing, that is, the cyclic expulsion and later accretion of the Interstellar Medium (Davies \& Phillipps 1988). Alternatively, interactions and/or mergers have been proposed as the mechanism responsible for triggering the star formation in dwarfs. From optical surveys (Campos-Aguilar. Moles, \& Masegosa 1993; Telles \& Terlevich 1995; Telles \& Maddox 2000) it was first concluded that gravitational interactions with optically bright galaxies are too rare to play a relevant role. However, studies at radio wavelengths (Taylor et al. 1993, 1995, 1996) established that H II/BCDs do have companions, most of them gas-rich, faint objects, which may go undetected in the optical. In agreement with these findings, more recent optical studies that extended the searches at lower masses and luminosities also found that a substantial fraction of SF dwarfs possess low mass companion galaxies (Noeske et al. 2001; Pustilnik et al. 2001). Ostlin et al. (2001) and Bergvall \& Ostlin (2002) put forward the idea that a merger between two galaxies with different metallicities, one gas-rich and one gaspoor, or infall of intergalactic clouds, could be the most plausible explanation for the starburst activity, at least in the most luminous BCDs. Recent studies, focused on individual objects, have shown that interactions do play a decisive role in the evolution of these systems (Johnson et al. 2004; Bravo-Alfaro et al. 2004, 2006).

Much work must still be done to elucidate the star formation histories of BCDs. The first step is to derive the properties of their stellar populations, quite a difficult task in such complex objects. The great majority of them cannot be resolved into stars, and the only way to study their stellar populations is by comparing their integrated properties with the predictions of evolutionary synthesis models. At any location in the galaxy, the emitted flux is the sum of the emission from the local starburst, the flux produced by the nebula surrounding the young stars, and the emission from the underlying, old stellar population, all possibly modulated by dust (Cairós 2000; Cairós et al. 2002, =C02; Cairós et al. 2007, =C07).

Another issue that was recently brought into discussion is the possible heterogeneity of the BCD class. Among the galaxies classified as BCDs we find a wide variety of objects spanning a large interval in luminosities $(-21.5<$ $\left.M_{B}<-14.0\right)$ and morphologies. Loose \& Thuan $(1986)$ developed a classification scheme for BCDs based on the appearance of the starburst and the shape of the external envelopes, distinguishing four subclasses: $i E$ galaxies, which show a complex inner structure with several $\mathrm{SF}$ regions over-imposed on an outer regular envelope; $n E$, objects with a clearly defined nucleus and regular isophotes; $i I$, which have irregular outer and inner parts and, finally, $i O$ galaxies, in which an outer envelope is not found. C01b have grouped the BCDs in four categories according to the position and morphology of the SF knots: nuclear starburst, which are galaxies with a prominent central starburst; extended starburst, galaxies with star formation spread over the entire galaxy, chain starburst, objects in which the SF knots are aligned to form a chain and finally, cometary starburst, galaxies with a "cometary" appearance, that is the star formation concentrated in one side of the galaxy.

This finding introduces an additional complication, be- cause it opens the possibility that different star formation mechanisms may operate in BCDs and that galaxies classified as BCDs may actually have different star formation histories and evolutionary paths.

Spectrophotometric studies that put together high quality data in a large wavelength range are fundamental to get insights into the star-formation process and history of BCDs. However, very few such studies have been carried out so far, and all of them focused on individual galaxies (Papaderos et al. 1999; Gil de Paz et al. 2000; C02; Guseva et al. 2003a,b. c; C07). These studies rely on conventional observing techniques: they combine good resolution broad-band frames in different bands (to spatially isolate the different stellar populations and map the dust distribution), with narrow-band imaging (needed to map the gas distribution, isolate the starburst regions and to derive their physical properties) and spectroscopy data (to derive the internal extinction, compute physical parameters and chemical abundances of the gas, and remove the contribution from emission lines); a sequence of long-slit spectra sweeping the regions of interest is usually taken. Therefore, these analyses require a great amount of observing time; besides, varying instrumental and atmospheric conditions make combining all the data together quite complicated. Long-slit spectroscopy has the additional problem of the uncertainty on the exact location of the slit.

We have thus undertaken a project to carry out an Integral Field Spectroscopy (IFS) mapping of a large sample of BCD galaxies. IFS is the ideal observational technique to study BCDs: each single exposure contains both spatial and spectral information in a large area of the galaxy, so just in one shot we collect information for all the SF regions as well as for the low surface brightness (LSB) stellar component. Besides, the kinematical information also allows us to investigate what mechanisms ignite the star-formation in BCDs. In terms of observing time, IFS observations of BCDs are an order of magnitude more efficient than traditional observing techniques, and provide simultaneous data for all spatial resolution elements under the same instrumental and atmospheric conditions, which guarantees a greater homogeneity of the dataset.

Here we present the first results from this project: we study five galaxies representative of the group of BCDs populating the high luminosity and metallicity range, and having rather complex morphologies. These objects, often referred to as Luminous Blue Compact galaxies (LBCGs, see Ostlin 1998; Kunth \& Ostlin 2000; $\mathrm{C} 01 \mathrm{~b})$ have a special significance in the general scenario of galaxy formation and evolution, as they are claimed to be the local counterparts of the luminous, compact, SF galaxies detected at higher redshifts (Garland et al. 2004). The proximity of these systems allows us to study their structure, stellar populations, star formation processes and chemical abundances with an accuracy and spatial resolution that cannot be achieved at intermediate/high redshift.

\section{OBSERVATIONS AND DATA REDUCTION}

\subsection{The Galaxy Sample}

In this pilot study we focus on five luminous galaxies $\left(M_{B} \leq-17\right)$ with complex morphologies and where 
star-formation takes place in a number of high surface brightness regions distributed atop an underlying, more regular host envelope (C01b). The galaxies were selected from the larger sample of 28 BCDs already studied by our group $(\mathrm{C} 01 \mathrm{a}, \mathrm{b})$. On one hand, these bright systems are the most suitable for a pilot study; on the other hand, in such objects, in which the star formation activity spread over the entire galaxy, an IFS mapping is essential.

Following the C01b classification, two galaxies (Mrk 297 and III Zw 102) belong to the extended starburst group and three (Mrk 370, Mrk 35 and Mrk 314) to the chain/aligned starburst class.

The basic data for the sample galaxies are given in Table $1{ }^{1}$. Column 5 lists the distances computed assuming a Hubble flow, with a Hubble constant $H_{0}=75$ $\mathrm{km} \mathrm{s}^{-1} \mathrm{Mpc}^{-1}$, after correcting recession velocities relative to the centroid of the local group for Virgocentric infall. Absolute magnitudes (column 6) were obtained from the $B$ asymptotic magnitudes (C01b), using the distances tabulated in column 5 . Notice that the asymptotic magnitudes listed in $\mathrm{C} 01 \mathrm{~b}$ were corrected for Galactic extinction following Burstein \& Heiles (1984); here they are recomputed using the Schlegel, Finkbeiner, \& Davis (1998) extinction values.

\subsection{Observations}

The data analyzed in this paper were obtained in two observing runs. The first run took place in 1999 August, and the second in 2003 February, both using the William Herschel Telescope (WHT), at the Observatorio del Roque de los Muchachos (ORM) on the island of La Palma.

The WHT was used in combination with the INTEGRAL fiber system (Arribas et al. 1998) and the WYFFOS spectrograph (Bingham et al. 1994). INTEGRAL links the Nasmyth focus of the WHT with the slit of WYFFOS via three optical fiber bundles. These three bundles have different spatial configurations on the focal plane and can be interchanged on-line depending on the scientific program or the prevailing seeing conditions. At the focal plane, the fibers of each bundle are arranged in two groups, one forming a rectangle, and the other an outer ring mapping the sky. The data discussed in this paper were obtained with the standard bundles 3 and 2 (STD3 and STD2 hereafter). STD3 consists of 135 fibers, each 2.7 in diameter on the sky, 115 fibers forming a central rectangle and covering an area of $33^{\prime \prime} .6 \times 29^{\prime \prime} 4$ on the sky, and the other 20 fibers forming a ring $90^{\prime \prime}$ in diameter. STD2 has 219 fibers (189 science and 30 sky fibers), each 0 . 9 in diameter and covering a field of view of $16^{\prime \prime} \times 12^{\prime \prime}$.

In 1999 August the WYFFOS spectrograph was equipped with a 600 groove $\mathrm{mm}^{-1}$ grating and a Tek CCD array of $1124 \times 1124$ pixels $24 \mu \mathrm{m}$ in size. The night was non-photometric and the seeing about $1^{\prime \prime} 3$. In February 2003 observations of Mrk 35 were done using the 1200 groove $\mathrm{mm}^{-1}$ grating and the same Tek CCD. We observed again in non-photometric conditions, with a seeing of about $1^{\prime \prime} .5$.

A complete log of the observations is provided in Table 2 .

Complementary broad and narrow-band $(\mathrm{H} \alpha)$ images are also available. These images were taken in different

${ }^{1}$ A collection of color and $\mathrm{H} \alpha$ maps of the galaxies can be found at: http://www.iac.es/proyect/GEFE/BCDs/ observing runs, using various telescopes and instrumental configurations. Details about the observations and the data reduction process can be found in C01a,b and C07.

\subsection{Data Reduction and Analysis}

The data reduction consists of two main steps: i) basic reduction of the spectra (i.e. bias, flatfielding, wavelength calibration, etc.) and ii) generation of spectral feature maps (e.g. line intensities, radial velocity, etc).

The first step was performed in IRAF, following the standard procedures used in fiber data processing (see e.g. García-Lorenzo et al. 1999). We obtained typical wavelength calibration errors of $0.1 \AA$, which give velocity uncertainties of \pm 6 and $\pm 4.5 \mathrm{~km} \mathrm{~s}^{-1}$ for [O III] $\lambda 5007$ and $\mathrm{H} \alpha$, respectively. We corrected the spectra for differential atmospheric refraction effects following the method proposed by Arribas et al. (1999); the differential atmospheric refraction was estimated according to the model given by Allen (1973). In those cases in which the air mass was close to one, such correction was not necessary.

For the generation of the spectral features maps, a two-dimensional interpolation was applied using the IDA package García-Lorenzo, Acosta-Pulido, \& Megías-Fernández 2002). In particular we transformed an ASCII file with the actual position of the fibers and the spectral feature corresponding to each fiber into a regularly spaced rectangular grid. In this way we built up images of $70 \times 70$ pixels with a scale 0.5 pixel $^{-1}$ for data from STD3, and $67 \times 67$ pixels with a scale $0 . \prime 3$ pixel $^{-1}$ for data from STD2. These images can then be treated with standard astronomical software packages. Maps generated in this way are presented in section 3 and beyond.

As mentioned before, observations were done under non-photometric conditions, and no spectrophotometric stars were observed, so no absolute flux calibration could be done. However, a relative calibration, aimed at computing the correct line flux ratios, was carried out by means of the spectral data published by Moustakas \& Kennicutt (2006), which lists line fluxes integrated over a large area of the galaxies (all of our 5 objects are included in their sample).

We proceeded as follows. First, for each object we obtained a "total" spectrum by adding up the spectra of all the science fibers, and computed the ratios, in counts, between the $\mathrm{H} \beta$, [O III] $\lambda 5007$, [N $\mathrm{II}] \lambda 6584$, [S II] $\lambda \lambda 6717,6731$ lines and $\mathrm{H} \alpha$. We then compared these values to the line ratios derived from the Moustakas \& Kennicutt (2006) data, and determined, for each such ratio, and for each object, the factor to convert count ratios into flux ratios. For each line ratio, the final conversion factor was obtained by averaging out the corresponding values for each object, and the associated uncertainty estimated from its scatter, after taking into account measurement errors (see notes in Table 3). This method relies on the assumption that, while the field of view of INTEGRAL is a fraction of the area covered by Moustakas \& Kennicutt (2006) spectra, it includes most of the galaxy light, and thus area-related differences in line ratios are negligible.

\section{RESULTS}




\subsection{The spectra}

As a first step in the spectroscopic analysis, we identify and select a number of spatial regions for each galaxy by using high resolution continuum and $\mathrm{H} \alpha$ images already analyzed by our group (see C01b and C07). The selected regions include the galaxy nucleus (defined as the peak in the continuum emission), and the most luminous SF knots; for all the galaxies, we also produce what we call the LSB spectrum, obtained by adding up all the fibers spectra outside the nucleus and the brighter knots; this spectrum, free from strong gas emission, is more suitable for searching for absorption features.

By direct comparison of our reference images and INTEGRAL maps, we identify those fibers that cover the area of interest and create the final spectrum for each region by summing them. Figures [1] to [5] display the selected regions in each galaxy and their spectra.

The dominating features in the final spectra are the emission lines $(\mathrm{H} \beta,[\mathrm{O}$ III $] \lambda 5007, \mathrm{H} \alpha,[\mathrm{N} \mathrm{II}] \lambda 6584$ and [S II] $\lambda \lambda 6717,6731)$, but significant differences appear both among the objects, and among different regions in a same galaxy. In particular, several regions show a very high continuum, with strong absorption features (indicating the presence of a substantial contribution of old stars) whereas other regions display a flat spectrum, with prominent emission lines and no absorption features, characteristic of a dominant OB population. The shape of the continuum, the strength of the emission and absorption features or the presence of several lines strongly vary across the objects. A more detailed description of the spectra of each galaxy is provided below:

- Mrk 370 - We define five spatial regions (see Figure 11): the nucleus, the two major $\mathrm{H} \alpha$ knots (R1 and $\mathrm{R} 2$ ), the smaller $\mathrm{H} \alpha$ knots at the north (R3) and the LSB component. The spectra of the nuclear region and $\mathrm{R} 2$ show a high continuum and strong absorption wings around $\mathrm{H} \gamma$ and $\mathrm{H} \beta$, indicating the presence of a substantial fraction of older stars; the absorption lines Mg I $\lambda \lambda 5167,5173$ and Na I D $\lambda 5892$ are detected. In R3, with weaker gas emission, the absorption features are more evident. R1, on the contrary, has a flat continuum, with no absorption features, characteristic of a population of young stars. Mg I $\lambda \lambda 5167,5173$ and $\mathrm{Na}$ I D $\lambda 5892$ are visible in the LSB component spectrum.

- Mrk 35 - This galaxy was observed with a different configuration, which provides higher spectral resolution in a smaller wavelength range. Five regions have been defined (see Figure 21). INTEGRAL does not allow to resolve the central knots, which we lump together within the aperture R1; the nucleus (continuum peak) does not coincide with any $\mathrm{H} \alpha$ peak. Both the nucleus and $\mathrm{R} 1$ have flat spectra with prominent emission lines, in which [O I] $\lambda \lambda 6300,6363$, He I $\lambda 5876$, He I $\lambda 6678$ and [S III] $\lambda 6312$ are also visible. $\mathrm{R} 2$, which includes several smaller SF regions towards the NE, and R3, which corresponds with the two knots detached from the main body of the galaxy, both display similar flat spectra. No absorption lines are visible in the spectrum of the LSB component.
- Mrk 297 - We study seven SF regions, plus the nucleus and the LSB emission (see Figure 3). The characteristics of the spectra in the selected regions differs significantly. The nucleus, which does not coincide with any $\mathrm{H} \alpha$ peak, shows a very high continuum, and displays strong $\mathrm{H} \gamma$ and $\mathrm{H} \beta$ absorption lines; R1 and R2 have a flat continuum, with no evident absorption features; both spectra show He I $\lambda 5969$, but only in $\mathrm{R} 2[\mathrm{O} \mathrm{I}] \lambda 6300$ is apparent. $\mathrm{R} 3$, located in the central region of the galaxy, has a higher stellar continuum, significant absorption features, and strong He I $\lambda 5969$ and [O I] $\lambda 6300$ lines. R4, R5, R6 and R7, show flat spectra; [O I] $\lambda 6300$ is detected in all these regions except R7. The signal-to-noise in the LSB spectra is not large enough to search for absorption features.

- Mrk 314 - This galaxy was observed with the STD2 of INTEGRAL, which provides higher spatial resolution in a smaller field of view. Our IFU data allow us to resolve the five larger knots detected in the $\mathrm{H} \alpha$ frames (Figure 4). The nucleus, which coincides with the $\mathrm{H} \alpha$ knot $\mathrm{R} 1$, displays a nebular spectrum dominated by emission lines; [O I] $\lambda \lambda 6300,6363$, He I $\lambda 5876$ and He I $\lambda 6678$ are visible and $\mathrm{Mg}$ I $\lambda 5167$ is detected in absorption. The five selected regions show flat spectra, typical of $\mathrm{H}$ II regions. No absorption features are detected in the spectrum of the LSB component.

- III Zw 102 - We study four spatial regions besides the nucleus and the LSB component (Figure 5). The nucleus does not coincide with any $\mathrm{H} \alpha$ peak and has a very high continuum, with prominent absorption features (e.g. Mg I $\lambda 5167$, Na I D $\lambda 5892$ and absorption wings around $\mathrm{H} \beta$ ); interestingly, there is very weak [O III] $\lambda \lambda 4959,5007$ in emission. The spectra of the four selected regions are quite similar: all are dominated by a high continuum, have faint [O III] $\lambda \lambda 4959,5007$ lines and display pronounced absorption features. Indeed, the shape of the spectra resembles those typical of spiral galaxies, rather than of BCDs.

In Appendix we present the individual spectra corresponding to each fiber in a selected spectral interval centered around $\mathrm{H} \beta$ (in the case of Mrk 35, around $\mathrm{H} \alpha$ ).

\subsection{Emission line ratios}

We measured the intensity of the lines in the spectrum of each region defined in the previous section. The emission line profiles were fitted by a single Gaussian function using the DIPSO package inside the STARLINK environment $^{2}$. In order to obtain accurate values of the Balmer emission line fluxes, we took the underlying stellar absorption into account by fitting two Gaussians in those cases where absorption wings are visible. The $\mathrm{H} \alpha+[\mathrm{N} \mathrm{II}] \lambda \lambda 6548,6584$ lines were fitted simultaneously, imposing that the three lines have the same velocity shift and the same width, and fixing to 3 the ratio between the two nitrogen lines. Also the [O III] $\lambda \lambda 4959,5007$ lines

\footnotetext{
2 http://www.starlink.rl.ac.uk/
} 

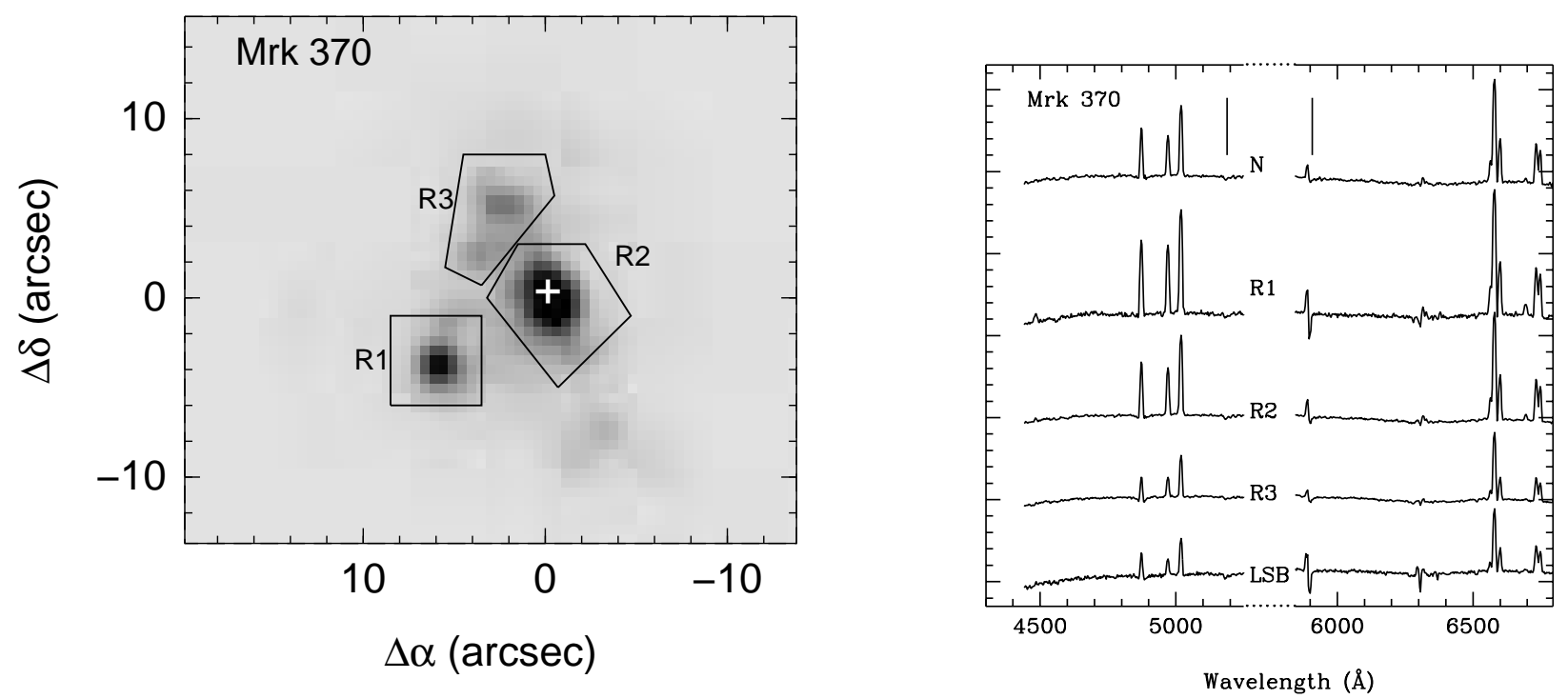

FIG. 1. - Left: Continuum subtracted $\mathrm{H} \alpha$ image of Mrk 370 from C01b; the different regions selected in the galaxy are labeled. The white cross marks the galaxy nucleus. The area shown is the INTEGRAL field of view. Right: Representative spectra of the regions selected for analysis. The horizontal axis does not represent a continuous range in wavelength, but two sub-intervals around $\mathrm{H} \beta$ and [O III] $\lambda \lambda 4959,5007$ and around $\mathrm{H} \alpha+[\mathrm{N} \mathrm{II}] \lambda \lambda 6548,6584$ and [S II] $\lambda \lambda 6717,6731$. For sake of clarity the spectra are shown in logarithmic scale and arbitrarily shifted on the $\mathrm{Y}$ axis. The step between small tickmarks is 0.2 dex. Two vertical lines mark the position of the Mg I and $\mathrm{Na} I \mathrm{D}$ absorption lines. $\mathrm{N}$ is the spectrum of the nucleus.

were fitted simultaneously, again fixing the line ratio to 3 , using the same width for both lines, and forcing them to have the same velocity shift. The same constraints (except the one on the flux ratio) were applied to the [S II] $\lambda \lambda 6717,6731$ doublet. The continuum was fitted with a linear or quadratic function.

The most relevant line ratios for each region are listed in Table 3 ,

In virtually all the regions, the $\mathrm{H} \alpha / \mathrm{H} \beta$ line ratio is considerably larger than the nominal values of 2.86 (Osterbrock \& Ferland 2006), indicating that they are significantly affected by dust obscuration. Particularly high values are found for Mrk 297 and III $\mathrm{Zw}$ 102. This result is in agreement with previous findings: the color maps published in $\mathrm{C} 01 \mathrm{~b}$ and $\mathrm{C} 03$ already pointed out to the presence of dust in both objects; the presence of strong dust lanes in III $\mathrm{Z}_{\mathrm{w}} 102$ has been long known (Demoulin 1969; Brosch \& Loinger 1991).

\subsubsection{Ionization Mechanisms}

The ionization mechanisms for the individual SF regions can be studied by means of various diagnostic diagrams (see the classical paper by Baldwin et al. 1981). Here we follow the scheme proposed by Veilleux \& Osterbrock (1987) in which the [O III] $\lambda 5007 / \mathrm{H} \beta$, [O I] $\lambda 6300 / \mathrm{H} \alpha,[\mathrm{N}$ II] $\lambda 6584 / \mathrm{H} \alpha$ and [S II] $\lambda \lambda 6717,6731 / \mathrm{H} \alpha$ line ratios are used to distinguish among different ionization mechanisms (radiation from young stars, shocks and AGNs).

The position of the different regions under study in these diagrams is shown in Figure 6 (Mrk 35 is not included because in this galaxy [O III] $\lambda 5007$ and $\mathrm{H} \beta$ are outside the observed wavelength interval). The empirical boundaries between the different zones (from
Veilleux \& Osterbrock 1987) as well as the theoretical boundaries proposed by Kewley et al. (2001) are also plotted. The main result emerging from this plot is that radiation from young stars is the dominant ionization mechanism in all the galaxies. Also, we found that all the different SF regions of a same galaxy lie very close to each other in the diagram: the SF knots identified in Mrk 314 occupy the upper zone (with [O III] $\lambda 5007 / \mathrm{H} \beta$ $\geq 3$ ), while the III $\mathrm{Zw} 102$ regions have lower excitation parameters $\left(\left[\begin{array}{ll}\mathrm{O} & \mathrm{III}\end{array}\right] \lambda 5007 / \mathrm{H} \beta \leq 1\right)$. In three galaxies, the nucleus unequivocally has a $\mathrm{H}$ II-like ionization. Only the nucleus of Mrk 297 falls on the boundary between the $\mathrm{H}$ II and the LINER regions in the [S II] $\lambda \lambda 6717,6731 / \mathrm{H} \alpha$ diagram.

\subsubsection{Electron Densities and Chemical Abundances}

From the measured emission line fluxes, we can derive electron densities and oxygen abundances in the selected galaxy regions.

As we did not detect the [O III] $\lambda 4363$ emission line in any of the spectra, we could not calculate the electron temperature (the alternative empirical calibrator proposed by Pilyugin (2001) to estimate $T_{\mathrm{e}}$ relies on the flux of the [O II] $\lambda 3727$ line, which is outside the observed spectral range). Therefore, in order to compute electron densities, we assumed an electron temperature of $10000 \mathrm{~K}$, a good approximation for most H II regions (Osterbrock \& Ferland 2006).

We determined the electron density of the ionized gas from the [S II] $\lambda 6717 / \lambda 6731$ ratio, using the task TEMDEN, based on the FIVEL program Shaw \& Dufour 1995), which is included in the IRAF package NEBULAR. We find densities in between $\leq 100$ and $360 \mathrm{~cm}^{-3}$, typical of classical H II regions (Copetti et al. 2000). In all 

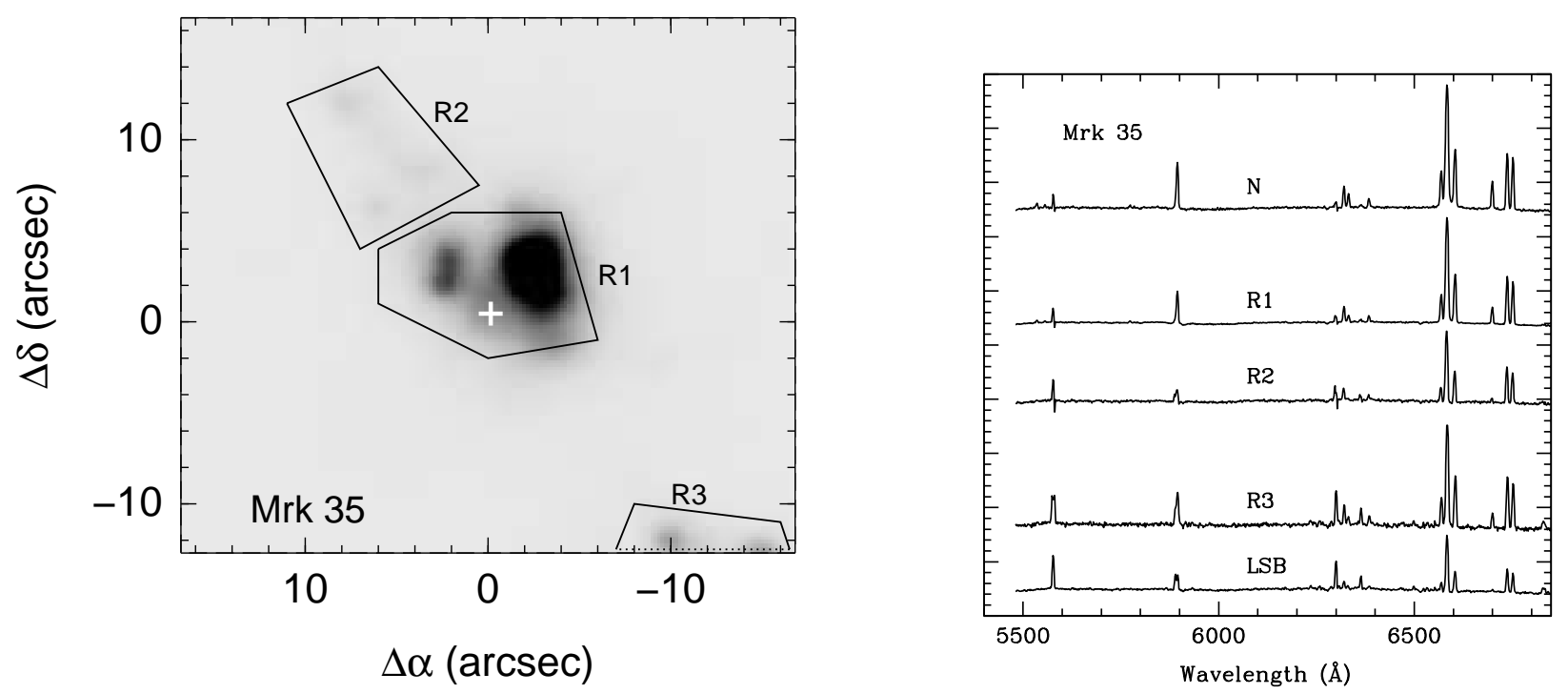

Fig. 2.- Same as in Figure1 for Mrk 35 (Continuum subtracted H $\alpha$ image from C07)
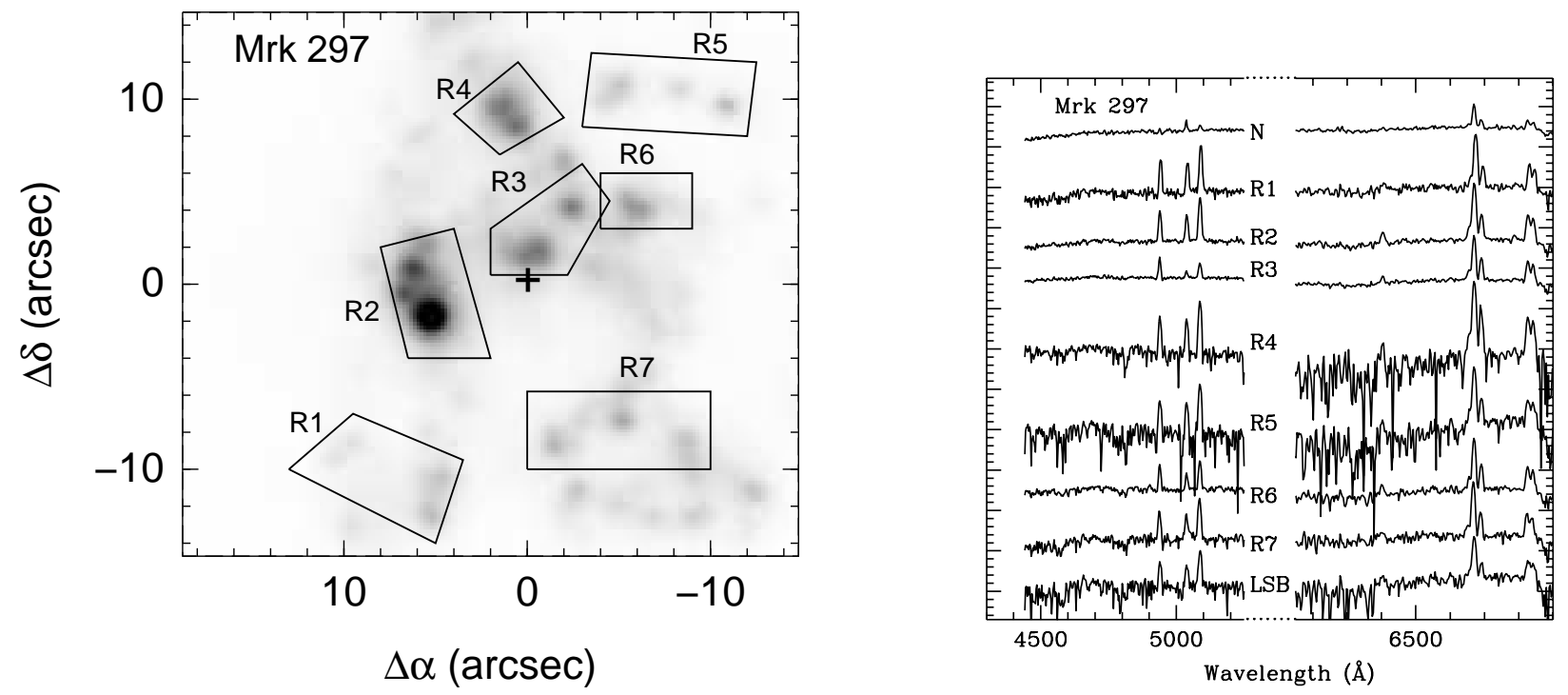

Fig. 3.- Same as in Figure 1 for Mrk 297. 
galaxies except Mrk 370, we find that the electron density significantly varies across the galaxy.

Abundances were estimated using the empirical calibrator N2 (Storchi-Bergmann et al. 1994), following the calibration made by Denicoló et al. (2002). The abundances range from $\simeq 0.3 Z_{\odot}$ to $\simeq 1.5 Z_{\odot}($ the solar value is taken as $12+\log (\mathrm{O} / \mathrm{H})=8.69$, Lodders 2003).

Electron densities and oxygen abundance values are listed in Table 4

\subsection{Intensity maps}

The procedure that we follow to generate the maps is described in Section 2.3. All maps are corrected for galactic extinction.

\subsubsection{Continuum and Line Emission Maps}

In order to study the properties of the stellar component, we build continuum maps within selected wavelength intervals free from emission lines, which trace the stellar properties ("pure continua"), e.g. 5600-5800 А or 7000-7200 А.

Figure 7 shows these continuum maps in the spectral interval 7000-7200 $\AA$ for all galaxies except Mrk 35, for which we display the continuum map in the interval 5600-5800 A. The location of the peak of the "pure continuum "maps does not depend on what specific "gasfree" spectral range is used; therefore, we can safely define the continuum peaks as the "optical nucleus" of the galaxies. Mrk 370, Mrk 297 and Mrk 314 have a second continuum maximum, which could be a secondary nucleus (see the discussion in Section 4).

$\mathrm{H} \alpha$ and [O III] $\lambda 5007$ line intensity maps are also displayed in Figure 7. For all galaxies, both $\mathrm{H} \alpha$ and [O III] $\lambda 5007$ maps have a similar morphology, as expected in objects ionized by stars, significantly different from the continuum maps. The emission line maps show several peaks distributed across the whole field of view, whereas the continuum distribution is more regular. Only in Mrk 314 the peak in the emission line maps spatially coincides with the peak in the continuum. In Mrk 297 and III Zw 102, the current star-formation activity is spread all over the mapped region.

\subsubsection{Line ratio maps}

Maps of the [O III $] \lambda 5007 / \mathrm{H} \beta,[\mathrm{N} \mathrm{II}] \lambda 6584 / \mathrm{H} \alpha$, and $\mathrm{H} \alpha / \mathrm{H} \beta$ ratios are presented in Figure 8 .

The [O III] $\lambda 5007 / \mathrm{H} \beta$ ratio (Figure 8, column 1), commonly used as an indicator of the excitation degree (McCall et al. 1985), shows a complex pattern in all the objects; the highest [O III] $\lambda 5007 / \mathrm{H} \beta$ values $(\geq 3)$ usually trace the line emission peaks, except in Mrk 297. The five galaxies have the excitation levels expected for $\mathrm{H}$ II-like regions in the whole field of view.

The morphology of the [N II] $\lambda 6584 / \mathrm{H} \alpha$ maps is similar to the [O III] $\lambda 5007 / \mathrm{H} \beta$ maps but, as expected, it follows the opposite trend: the lower [N II] $\lambda 6584 / \mathrm{H} \alpha$ values are reached in the center of the $\mathrm{SF}$ knots (where the $\mathrm{H} \alpha$ intensity peaks), while higher ratios are found in their surroundings. (This behavior towards higher excitation degree when the intensity is larger can be related to the decreasing distance from the young ionizing cluster.)

The outer regions of III Zw 102 have [N II] $\lambda 6584 / \mathrm{H} \alpha$ values larger than those expected in $\mathrm{H}$ II-regions
([N II $] \lambda 6584 / \mathrm{H} \alpha>0.6)$, which may suggest the presence of shock-like excitation mechanisms in the circum-knots environment.

The $\mathrm{H} \alpha / \mathrm{H} \beta$ maps all show a highly inhomogeneous structure, with strong variations of the extinction across the galaxy. Just assuming a single, spatially constant value for the extinction (for instance obtained from longslit spectra and thus dominated by the nuclear/brighter knot emission) can lead to large errors in the derivation of the magnitudes and fluxes of the knots, and hence in the determination of the star-formation rate and the properties of the stellar populations (see also Kehrig et al. 2007).

\subsection{Kinematics of the Ionized Gas}

The central wavelength of the Gaussians fit to the lines in the individual spectra gives us the radial velocity of the ionized gas at each observed position. (The spectral resolution of our data, about $9.5 \AA$ FWHM, prevents us from deriving reliable velocity dispersions).

Figure 9 displays the velocity field of the ionized gas derived from the [O III] $\lambda 5007$ (column 1) and the $\mathrm{H} \alpha$ (column 2) emission lines (for Mrk 35 we used $\mathrm{H} \alpha$ and [S II]). In Table 5] we present the velocity at the position of the optical nucleus derived from [O III] $\lambda 5007$ and $\mathrm{H} \alpha$ emission lines, as well as the peak-to-peak velocity difference in the central 5 arcsec of the galaxies.

In general, the velocity maps are irregular, making their interpretation difficult. In all five galaxies the velocity field of the inner regions are inconsistent with ordered motions, albeit in three galaxies we also find a large scale behavior of receding and approaching velocities. Although the [O III] $\lambda 5007$ and the $\mathrm{H} \alpha$ velocity maps are in broad agreement, there are some differences, which may reflect the fact that low $(\mathrm{H} \alpha)$ and high-ionization ([O III $] \lambda 5007$ ) lines trace the kinematics of two different gaseous components. Observational evidences (Mulchaev et al. 1992; Arribas et al. 1997; Márquez et al. 1998; García-Lorenzo et al. 2005) suggest a general trend for the low-ionization gas to be a better tracer of the general dynamics of the galaxies, while the high-ionization gas may present a more irregular kinematical pattern associated with outflows, inflows or tidal induced motions.

Mrk 370 and Mrk 35 display a general velocity pattern indicative of an overall rotation; Mrk 370 shows similar velocities and amplitudes for both the high and the low ionization gaseous components. III $\mathrm{Zw} 102$, while displaying the largest differences between the [O III] $\lambda 5007$ and the $\mathrm{H} \alpha$ kinematics, also shows a velocity field resembling a rotational system. Finally, Mrk 297 and Mrk 314 show small differences between high and low-ionization gas: in these two systems, the velocity field is irregular in the whole mapped area.

\section{RESULTS AND DISCUSSION ON THE INDIVIDUAL GALAXIES}

\subsection{Mrk 370}

This is the only galaxy in the sample that clearly fits into the BCD luminosity range (with $M_{B}=$ -17.00). Deep broad-band optical and near-infrared (NIR) surface photometry was presented in C01a,b and Noeske et al. (2005) respectively, while C02 published a 

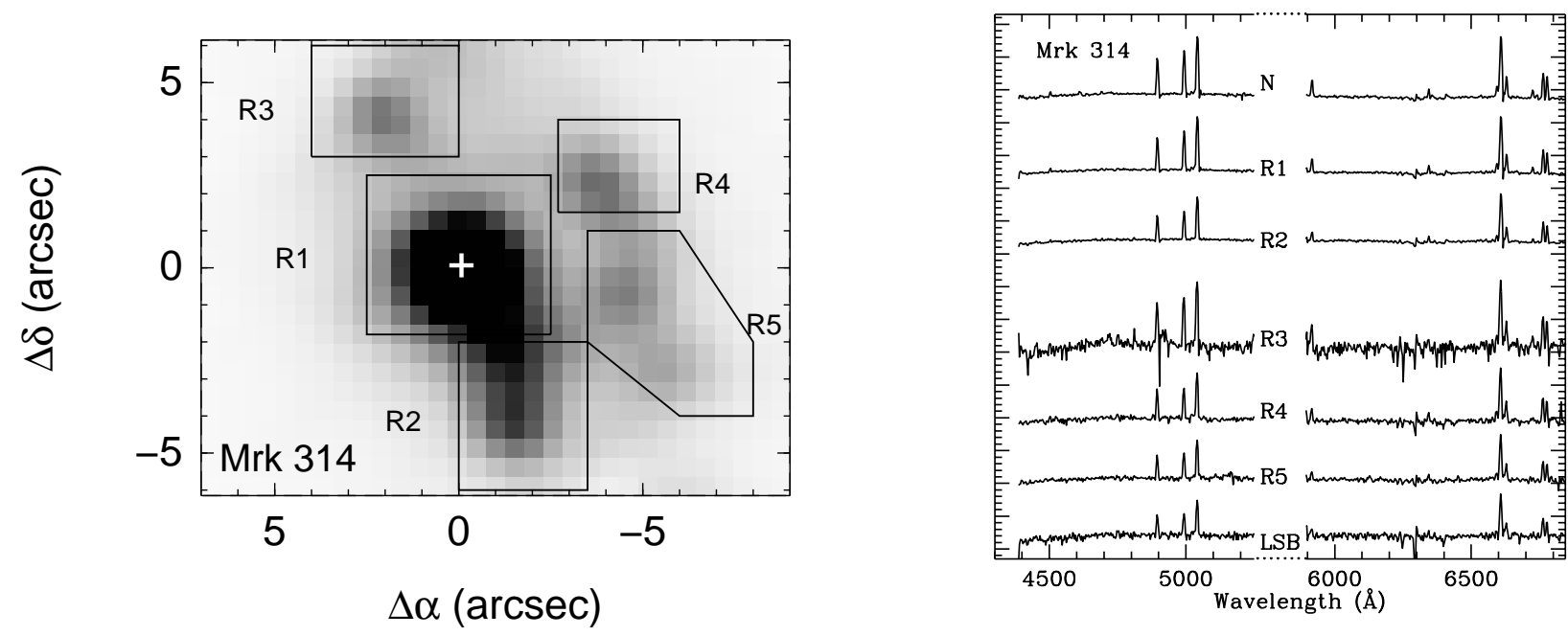

Fig. 4.- Same as in Figure 1 for Mrk 314.
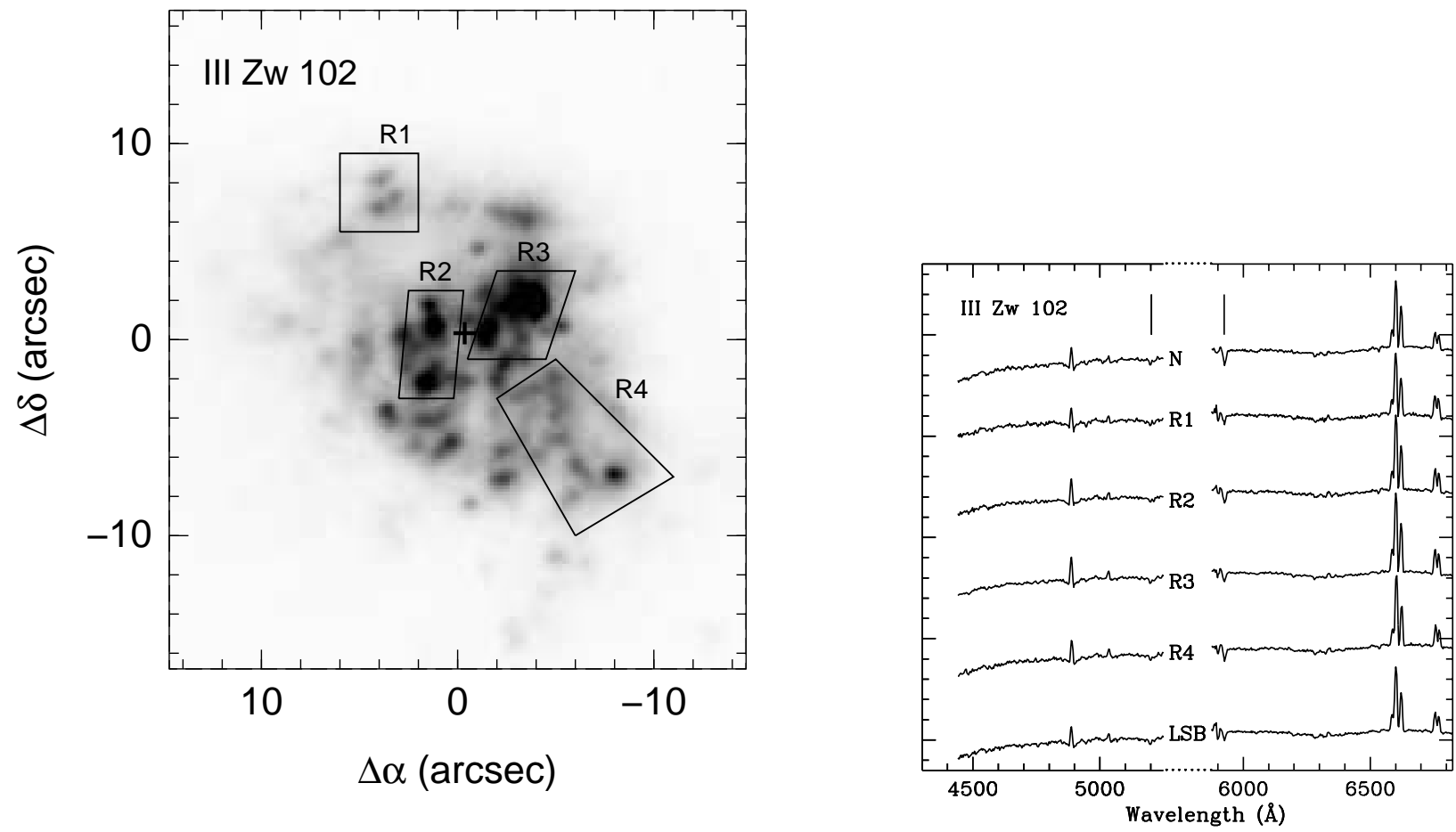

FIG. 5.- Same as in Figure 1 for III Zw 102. 


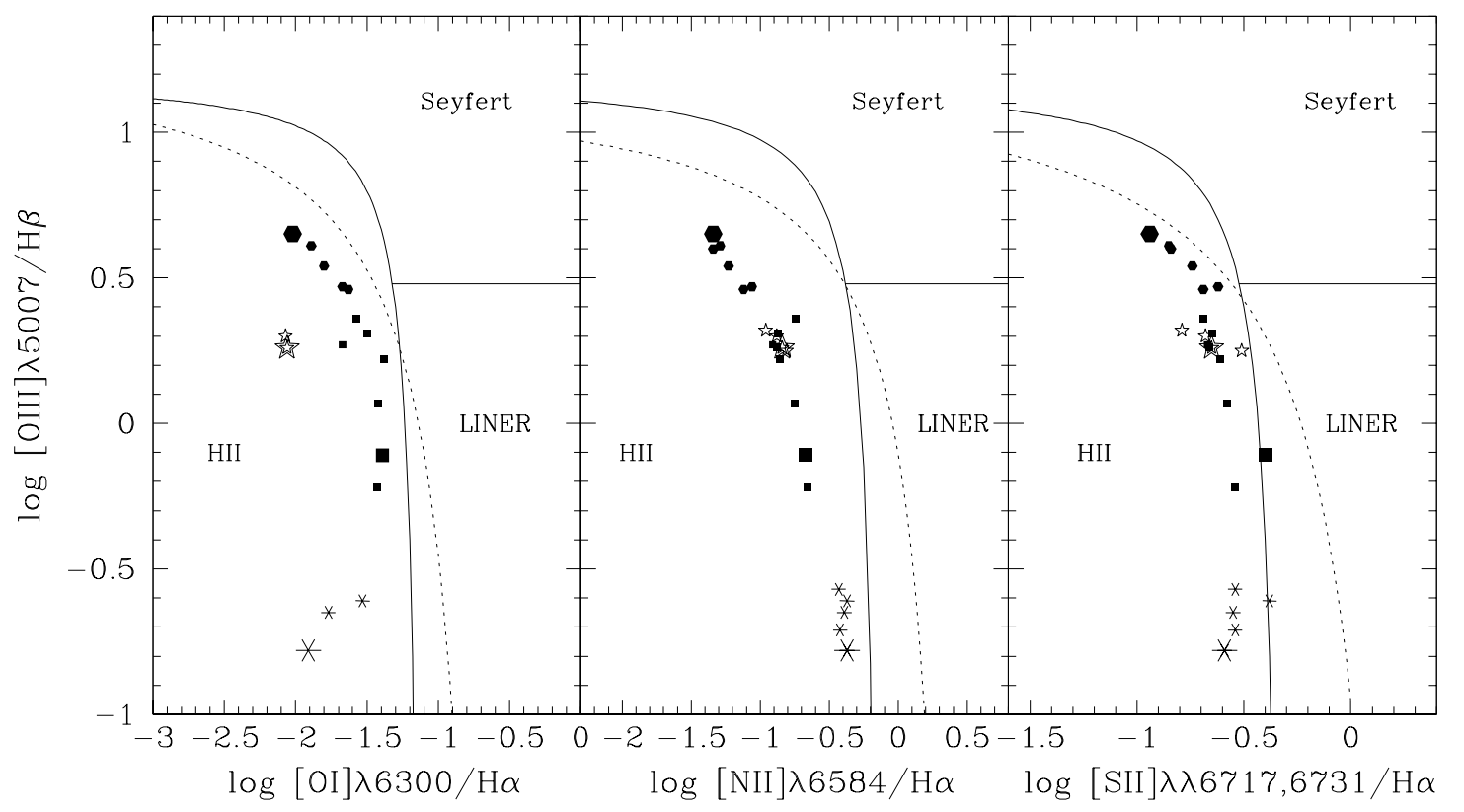

FIG. 6.- Optical emission line diagnostic diagrams for the selected regions in the sample galaxies. The curves separate Seyferts and LINERs from H II region-like objects; solid lines are the empirical borders from Veilleux \& Osterbrock (1987), while dotted lines represent the theoretical borders from Kewley et al. (2001). Different galaxies are shown with different symbols: stars, Mrk 370; squares, Mrk 297; hexagon, Mrk 314; asterisks, III Zw 102. The nuclei are shown with a larger symbol.

dedicated spectrophotometric study which included INTEGRAL data taken with the STD2 bundle. The galaxy shows two bright SF knots, located in the inner region, from which smaller knots emerge and form a structure resembling spiral arms. These two central knots were cataloged as "double nucleus" in Mazzarella \& Boroson (1993). The outer isophotes are regular and elliptical.

The current IFU data cover the central, brighter SF knots. We find similar values of the electron densities (in the low density limit) in the four selected regions, and constant oxygen abundances (within the uncertainties), slightly smaller than those reported in $\mathrm{C} 02$ $(12+\log (\mathrm{O} / \mathrm{H})=8.6$ in $\mathrm{R} 1$ and $12+\log (\mathrm{O} / \mathrm{H})=8.7$ in $\mathrm{R} 2$ ).

Continuum and emission line maps show similar morphologies, with the two central knots (R1 and R2) dominating the galaxy emission; $\mathrm{R} 2$ and $\mathrm{R} 3$ merge together in the continuum frames. $\mathrm{R} 1$, the peak in line emission, is not a strong continuum emitter. As already pointed out by $\mathrm{C} 02$ and Noeske et al. (2005), the large equivalent $\mathrm{H} \alpha$ width, the weak NIR flux, and the blue optical colors, all indicate that $\mathrm{R} 1$ is an $\mathrm{H}$ II region, and not the remnant of a captured galaxy, thus falsifying the double nucleus

\section{hypothesis.}

The [O III $] \lambda 5007 / \mathrm{H} \beta$ ratio reaches the highest values $(>4.0)$ in the emission peaks. The $[\mathrm{O}$ III $] \lambda 5007 / \mathrm{H} \beta$ and $[\mathrm{N} \mathrm{II}] \lambda 6584 / \mathrm{H} \alpha$ maps have similar morphologies, with [N II] $\lambda 6584 / \mathrm{H} \alpha$ dropping in those regions where [O III] $\lambda 5007 / \mathrm{H} \beta$ peaks. The $\mathrm{H} \alpha / \mathrm{H} \beta$ map shows an inhomogeneous pattern, in which the positions of the extinction peaks do not coincide with those of the emission line peaks.

Although the velocity fields derived from [O III] $\lambda 5007$ and from $\mathrm{H} \alpha$ are somewhat irregular, they suggest an overall rotation, with the rotation axis oriented southeast northwest, broadly aligned with the photometric minor axis of the $\mathrm{H} \alpha$ distribution.

\subsection{Mrk 35}

Mrk 35 also fits, albeit by a small margin, into the luminosity range of BCDs. Deep optical and NIR surface photometry was presented in C01a,b and C03, while a spectrophotometric analysis has been recently published by C07. The starburst knots of Mrk 35 are distributed in a bar-like structure aligned northeast-southwest. The brightest SF regions are located in the central part of the galaxy, in a "heart-shaped" configuration; a tail departs 

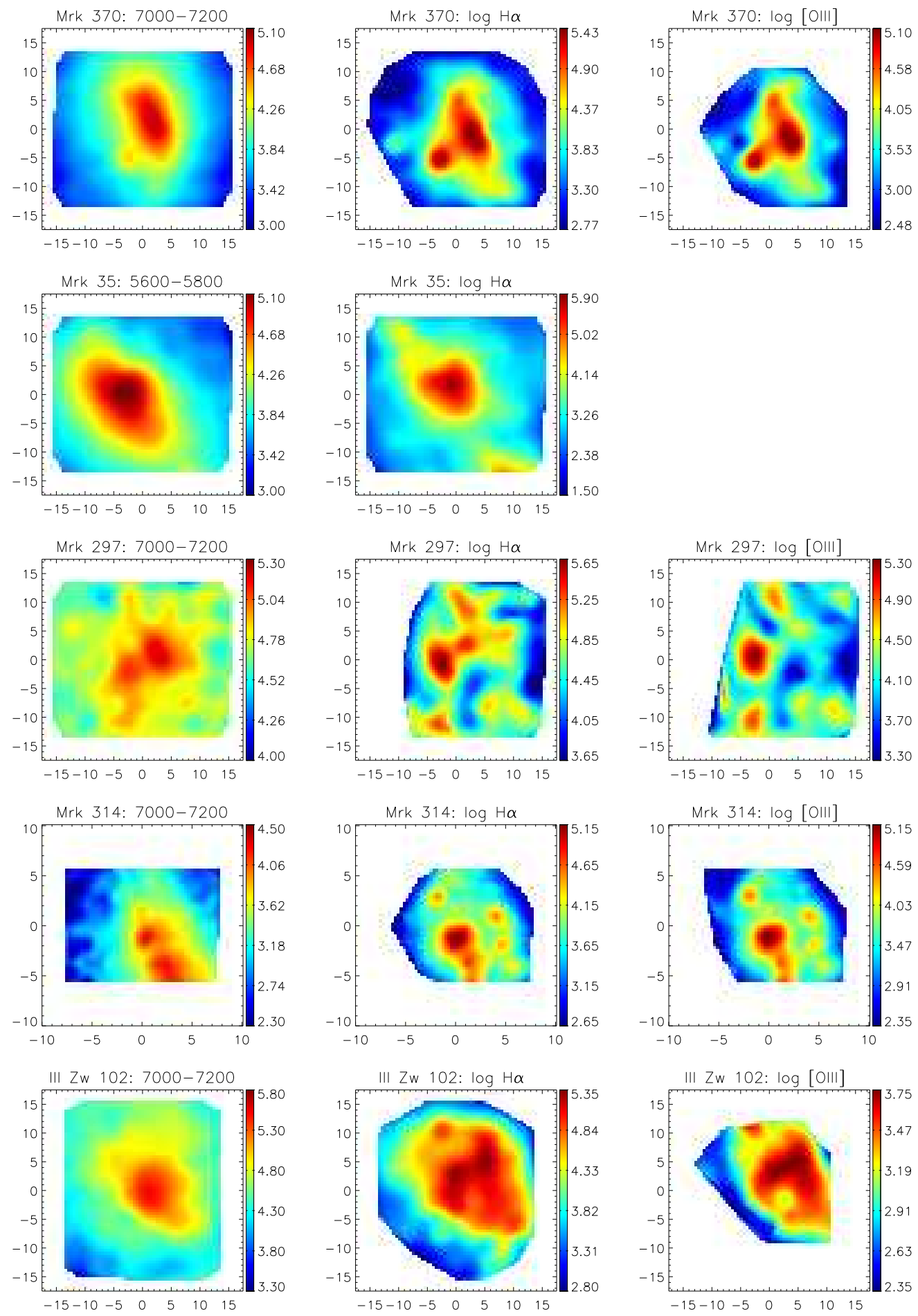

FIG. 7.- Two-dimensional distribution of the continuum emission derived by integrating the signal in the indicated spectral ranges free from emission line contribution ("pure continua"), and integrated line intensity maps derived by a Gaussian fit. Maps are shown on a logarithmic scale to bring up the fainter regions. Axis units are arcseconds; north is up, east to the left. 

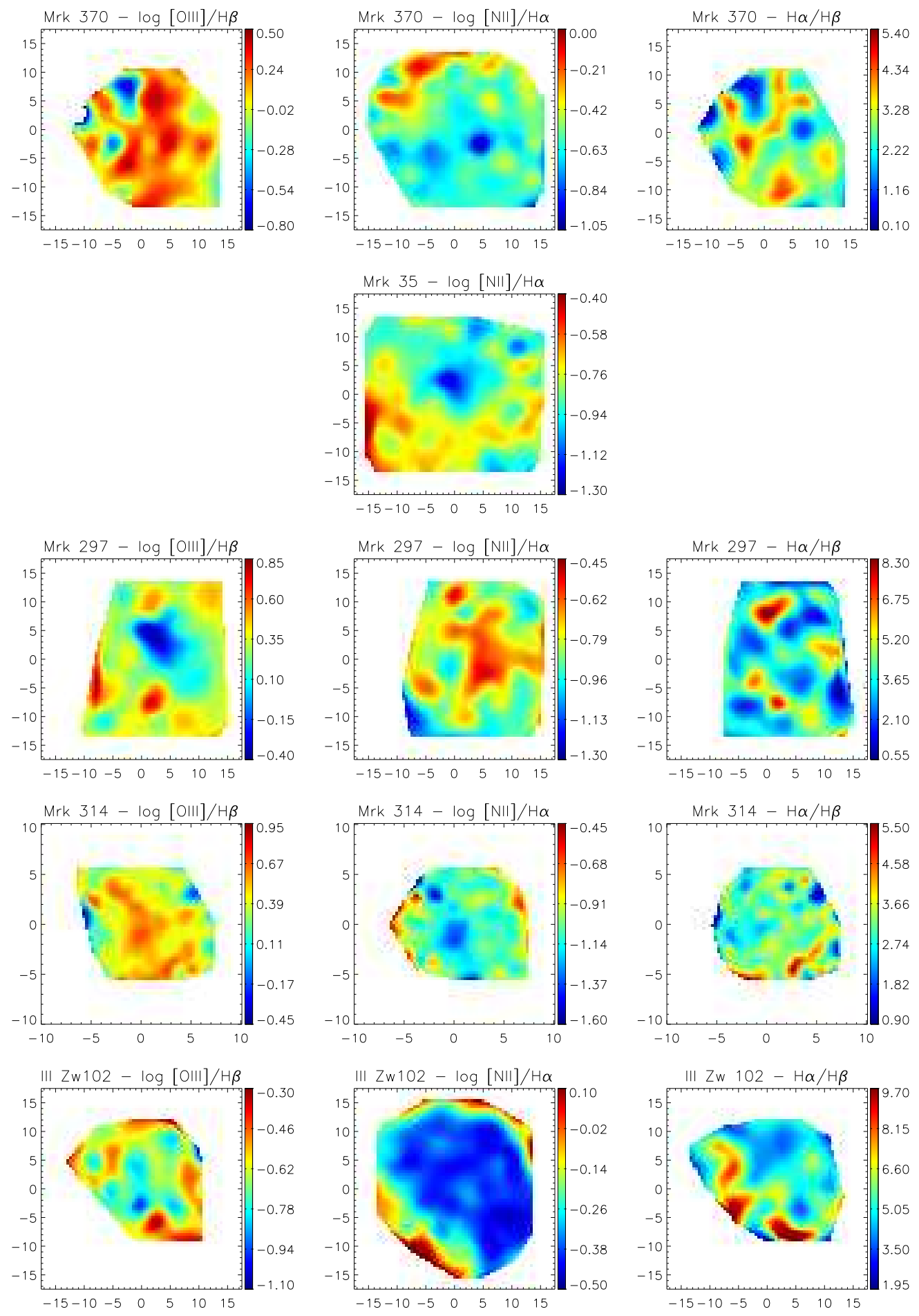

FIG. 8.- Ionization ratios [O III] $/ \mathrm{H} \beta$ and $[\mathrm{N} \mathrm{II}] \lambda 6584 / \mathrm{H} \alpha$, and extinction maps $(\mathrm{H} \alpha / \mathrm{H} \beta)$ for the observed galaxies.

from it towards southwest, connecting with two moderately bright knots $\sim 20^{\prime \prime}$ from the nucleus. The presence of Wolf-Rayet stars was reported by Steel et al. (1996) and $\mathrm{C} 07$. The starburst is placed atop a redder and regular envelope of older stars.

Our IFS data cover the central regions of the galaxy, the tail-like feature, and part of the two knots detected at the end of the tail. We find noticeable variations in the electron density, which peaks in the central, strongest
$\mathrm{H} \alpha$ knot (this knot contains about one half of the total $\mathrm{H} \alpha$ emission of the galaxy, C07). These findings, as well as the values found for the oxygen abundances, are in good agreement with the study by C07 based on longslit spectra.

With the present spatial resolution both continuum and emission line maps show a similar morphology. The individual star clusters seen by Johnson et al. (2004) are not resolved, and the emission peak in $\mathrm{H} \alpha$ is located $\sim 3^{\prime \prime}$ 

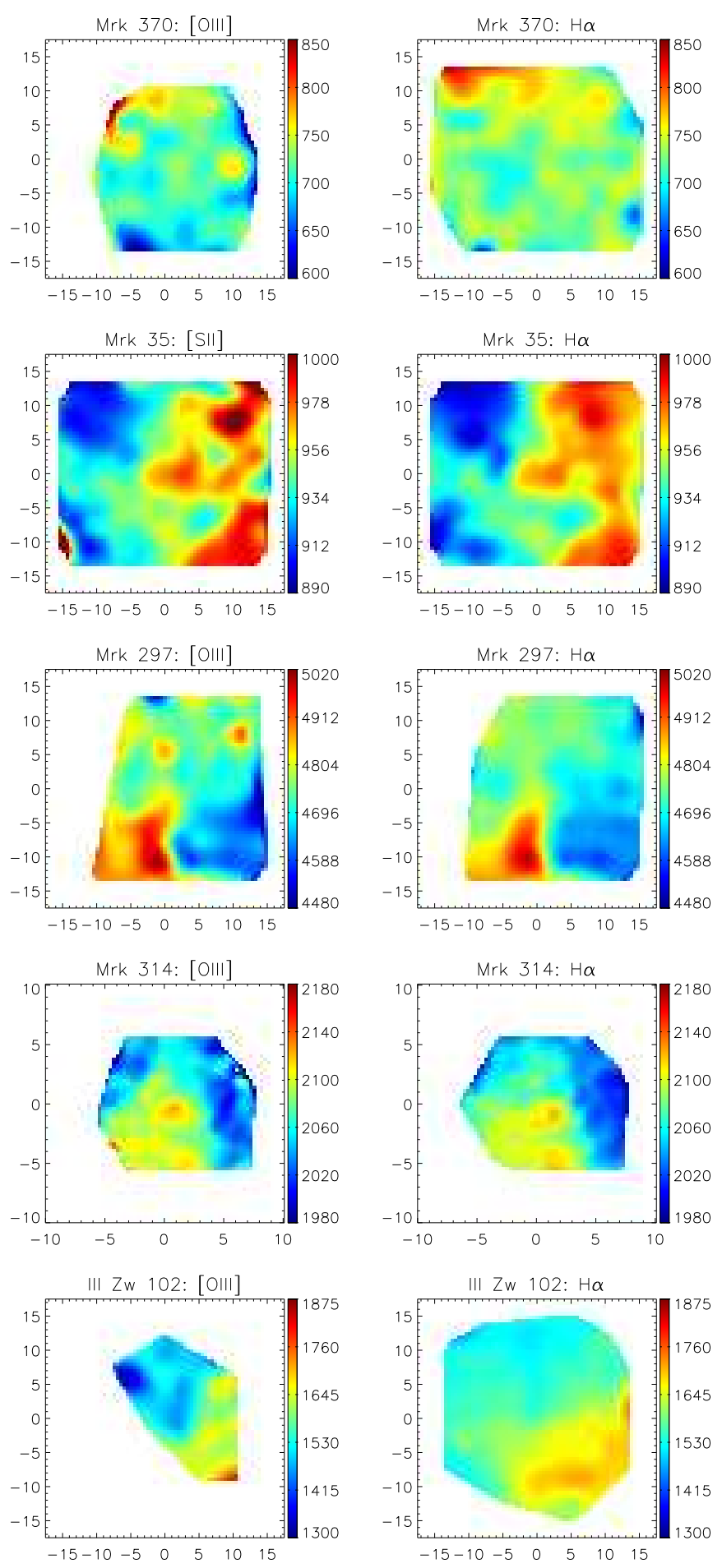

FIG. 9. - Velocity field of the ionized gas in the central region of the galaxies, for the [O III] $\lambda 5007$ ([S II] $\lambda \lambda 6717,6731$ for Mrk 35 ) and the $\mathrm{H} \alpha$ emission lines. Axis units are arcseconds; north is up, east to the left.

northwest of the continuum peak.

We could not compute the $[\mathrm{O}$ III $] \lambda 5007 / \mathrm{H} \beta$ nor the $\mathrm{H} \alpha / \mathrm{H} \beta$ ratio, as $[\mathrm{O} \mathrm{III}]$ and $\mathrm{H} \beta$ fall outside the spectral range. As expected in objects photoionized by stars, the [N II] $\lambda 6584 / \mathrm{H} \alpha$ map traces the star-formation regions, with a minimum in the central knot, R1. The small values of [N II] $\lambda 6584 / \mathrm{H} \alpha$ in the whole field of view indicate that shocks do not play a significant role.

The gas velocity field shows an overall regular rotation pattern, with the rotation axis aligned north-south (close to the galaxy minor axis, see $\mathrm{C} 07$ ), the west side receding and the east side approaching. However, in the central regions the velocity field displays a S-shaped distortion. This distorsion, also observed in the velocity profiles presented in $\mathrm{C} 07$, is suggestive of a counter-rotating component, located in the depression in gas emission between the central regions and the southwest knots. The velocity field is consistent with the hypothesis of an on-going 
minor merger, as proposed by Johnson et al. (2004).

\subsection{Mrk 297}

Mrk 297 is the most luminous galaxy in the sample; with $M_{B}=-21.05$, it is far from being a dwarf, but since its inclusion in the pioneering paper of Thuan \& Martin (1981), it is included in most BCDs samples. Mrk 297 has turned out to be the prototype of what are called today LBCG (see Kunth \& Ostlin 2000; Cairós et al. 2003; Garland et al. 2004). The galaxy is listed in the Atlas of Peculiar Galaxies (Arp 1966).

Optical and NIR surface photometry was presented in C01a,b and C03. The star formation takes place in numerous regions spread over nearly the entire system. $\mathrm{H} \alpha$ and continuum maps reveal a very complex morphology, suggestive of outflowing gas, with loops, tendrils and numerous filaments (see C01a,b and Martínez-Delgado et al. 2007). One remarkable feature is the double tail in the east side of the galaxy, extending out in the north-south direction. Martínez-Delgado et al. (2007) cataloged a total of 30 SF knots, for which they provided $\mathrm{H} \alpha$ photometry and colors. Ground-based $V-K(\mathrm{C} 03)$ and HST $V-R$ color maps (Papaderos 1998) indicate a large-scale, inhomogeneous absorption pattern. Mrk 297 has been studied extensively in the infrared, both with the Infrared Space Observatory (Metcalfe et al. 2005) and with the Spitzer Space Telescope (Whelan et al. 2007). With a total IR luminosity of $1.0 \times 10^{11} L_{\odot}$, it is classified as a luminous infrared galaxy (LIRG). Mrk 297 is thought to have arisen from the merger of two gasrich galaxies (Alloin \& Duflot 1979; Sage et al. 1993). Alloin \& Duflot (1979) identified the two peaks detected in the continuum frames as two compact cores embedded in a common envelope.

Our INTEGRAL data cover the whole starburst area, as well as a substantial fraction of the LSB component. We measure density variations across the galaxy, being the nucleus, R4 and R5 the regions with the highest values $\left(N_{\mathrm{e}} \geq 200 \mathrm{~cm}^{-3}\right)$. Such high densities could be explained by collisions between molecular clouds of the interstellar medium, or by gas inflow events. $\mathrm{R} 4$ and $\mathrm{R} 5$ have also the largest equivalent widths (Martínez-Delgado et al. 2007) implying that they are very young starbursts, probably ignited by the galaxy collision. Oxygen abundances are slightly larger in the nucleus and in R3; this abundance difference is consistent with the merger hypothesis. The values found for abundances $(8.46<12+\log (\mathrm{O} / \mathrm{H})<$ $8.64)$ are typical of late-type spiral galaxies, and much larger that those usually present in BCDs. The derived abundances are in good agreement with the values reported by Calzetti et al. (1994).

Continuum and emission line maps show different morphologies. In the continuum maps we clearly distinguish the two peaks identified by Alloin \& Duflot (1979) as the cores of the two merging systems, and a tail extending in the north-south direction. The emission line maps display a more knotty pattern; several very young regions are spread across the galaxy, the starburst having probably been triggered by the collision of the two systems. The emission line maps peak in $\mathrm{R} 2$, which includes one of the continuum maxima, while the continuum peak (that is, the galaxy nucleus) is close to but not coincident with the fainter knot $\mathrm{R} 3$.
Both the [O III] $\lambda 5007 / \mathrm{H} \beta$ and the [N II] $\lambda 6584 / \mathrm{H} \alpha$ excitation maps show an overall similar, complex morphology. The [O III] $\lambda 5007 / \mathrm{H} \beta$ minima (peaks in $[\mathrm{N}$ II] $\lambda 6584 / \mathrm{H} \alpha)$ are found in the central region (nucleus and $\mathrm{R} 3$ ), where there is decreased $\mathrm{H} \alpha$ emission. In the whole mapped area, both line ratios are inside the $\mathrm{H}$ II-region locus. The extinction pattern is also highly inhomogeneous, in agreement with the optical-NIR color map presented in $\mathrm{C} 03$.

The [O III] $\lambda 5007$ and $\mathrm{H} \alpha$ kinematics are in good agreement. The ionized gas velocity field shows chaotic rather than regular motions. The west side of the galaxy is less disturbed than the east side; a sharp velocity gradient in the EW direction is visible in the southern half of the map. This velocity field could be the result of the superposition of two colliding systems.

All in all, the morphological and kinematics results corroborate the interaction scenario proposed in previous works (Alloin \& Duflot 1979; Taniguchi \& Noguchi 1991). The double nucleus, the tail, the recent star formation, the large velocity gradients in the extra-nuclear regions and the perturbed gas in the whole field of view all are signs of a merger event.

\subsection{Mrk 314}

This is a LBCG, cataloged as a polar-ring galaxy candidate (Whitmore et al.|1990; van Driel et al. 2000) and also included in the Mazzarella \& Boroson (1993) catalog of multiple nuclei galaxies. Surface photometry in the optical and in the NIR was presented in C01a,b, Caon et al. (2005) and Noeske et al. (2005). Optical and NIR broadband images reveal three prominent peaks, aligned in the northeast-southwest direction. The narrow band images show that the star formation activity in the galaxy is distributed along the same direction, in a bar-like structure, which extends out to about $5 \mathrm{kpc}$ southwest of the nuclear region (Deeg, Duric, \& Brinks 1997; C01b).

The selected INTEGRAL configuration provides a higher spatial resolution, which allows us to resolve all the central knots, but a smaller field of view, which limits our study to the very central regions. The electron density peaks in the nucleus and in R5. The derived oxygen abundances are constant within the uncertainties, and are in good agreement with the values published in Shi et al. (2005).

Emission line and continuum maps display slightly different morphologies. The central knot, which is a moderate emitter in the continuum frames, is the peak in the emission line maps, whereas the peak in the continuum peak, south of the central knot, is a weak emission line emitter: this indicates that they have a different stellar content. Several SF knots, probably very young, are only visible in the emission line maps.

The $[\mathrm{O}$ III $] \lambda 5007 / \mathrm{H} \beta$ ratio map has a morphology similar to the emission line maps, and its peak coincides with the peak in $\mathrm{H} \alpha$ emission. The highest [O III] $\lambda 5007 / \mathrm{H} \beta$ value $(\geq 4)$ is reached in $\mathrm{R} 1$. Both the [O III $] \lambda 5007 / \mathrm{H} \beta$ and [N II] $\lambda 6584 / \mathrm{H} \alpha$ maps display basically the same pattern, and have values typical of $\mathrm{H}$ II-like ionization in the whole field of view. The $\mathrm{H} \alpha / \mathrm{H} \beta$ ratio map is irregular, and its features do not correlate with the ionized gas distribution. The higher values in the southernmost region are suggestive of a dust lane.

The velocity fields for [O III] $\lambda 5007$ and $\mathrm{H} \alpha$ have al- 
most identical pattern. The inner regions of Mrk 314 display a complex velocity field, which is in broad agreement with the one published in Shalyapina et al. (2004), which interpret it as the superposition of two kinematic subsystems of ionized gas.

\subsection{Zw 102}

This is another LBCG which, like Mrk 297, is included in many BCDs samples after the Thuan \& Martin (1981) classification, but does not comply with the luminosity criterion. The galaxy is listed in the Arp catalogue of Peculiar Galaxies (Arp 1966), and is also classified as Ep or SAap. It is an intriguing object, which shows regular external isophotes but a very clumpy morphology in its inner parts. Whitmore et al. (1990) considered III Zw 102 to be "related to polar-ring galaxies." Optical photometry was carried out by $\mathrm{C} 01 \mathrm{a}$; high resolution $\mathrm{H} \alpha$ maps were presented in $\mathrm{C} 01 \mathrm{~b}$ and Martínez-Delgado et al. (2007). The $\mathrm{H} \alpha$ emission is concentrated in the central region, and is resolved into numerous individual knots. There are plenty of holes, loops, and filamentary structures. Especially interesting are the large curvilinear structures that depart from the main body of the galaxy and extend out to $4 \mathrm{kpc}$ from the center. A strong dust lane is clearly visible in optical images and color maps (C01b).

Our INTEGRAL observations map the central starburst and part of the LSB component. The selected spatial regions present variations in the electron density, with the density peak being reached in the northern SF region, $\mathrm{R} 1$. As we pointed out in the case of Mrk 297, these density variations can be due to collisions among molecular clouds, or to gas inflows. All the SF knots have high oxygen abundances, with values typical of spiral galaxies. Interesting in this galaxy is the weak [O III] $\lambda 5007$ emission, indicating that the ionizing stars are not very young; also, the spectral shape is looks like that of a spiral galaxy, rather that resembling the flat spectrum of a BCD.

The continuum and the $\mathrm{H} \alpha$ maps have different morphologies. The continuum map shows a central maximum, that is the galaxy nucleus, while in line emission we see a more complex morphology, with two peaks located slightly north of the continuum peak, and a third, fainter peak further north.

The morphology of the $[\mathrm{O}$ III $] \lambda 5007 / \mathrm{H} \beta$ ratio resembles that of the $\mathrm{H} \alpha$ emission. [N II] $\lambda 6584 / \mathrm{H} \alpha$ have values larger than those typical for star-formation in the outer parts of the map; this could indicate that shocks are playing a significant role. The extinction map is inhomogeneous, and clearly shows the dust lanes detected by C01b.

The ionized gas velocity field shows an overall regular rotation pattern, around a kinematic axis roughly oriented ESE-WNW. The optical nucleus appears to be located on this axis.

\section{SUMMARY AND CONCLUSIONS}

We present results from what is, as far as we know, the first Integral Field Spectroscopy analysis of a sample of galaxies catalogued as Blue Compact Dwarfs.

- With the help of high resolution optical images, we define several regions of interest in each object, and for each of them we measure emission line fluxes and compute the most relevant line ratios. These data show that:

- The strength of emission lines and absorption features, the shape of the continuum, and the presence of other relevant lines significantly vary across each galaxy. This indicates varying physical conditions and/or stellar content.

- All the regions studied have H II-like ionization (i.e. star-formation).

- All the identified regions in the observed galaxies have low electron densities, ranging from $\leq 100$ to $360 \mathrm{~cm}^{-3}$, typical of classical $\mathrm{H}$ II regions. The electron density also shows significant spatial variations in four out of the five galaxies studied.

- The derived oxygen abundances are relatively large in all the cases, ranging from $Z_{\odot} \simeq 0.3$ to $Z_{\odot} \simeq 1.5$. No significant oxygen abundances variations within a same object are found.

- We build maps of the stellar continuum, emission line fluxes and excitation ratios, finding that:

- Continuum and emission line morphologies are generally different.

- The excitation ratios [O III] $\lambda 5007 / \mathrm{H} \beta$ and [N II] $\lambda 6584 / \mathrm{H} \alpha$ are typical of HII regions in the whole observed fields. Only in the outer regions of III Zw 102 the [N II] $\lambda 6584 / \mathrm{H} \alpha$ ratio may suggest the presence of shocks.

- All the galaxies present a complex extinction pattern. Assuming that the extinction coefficient is constant across the whole galaxy can lead to considerable errors in the derivation of magnitudes and colors and in the determination of the star formation rate and ages.

- The $\mathrm{H} \alpha / \mathrm{H} \beta$ ratios are higher that the theoretical values, indicating the presence of significant amounts of dust in these galaxies.

- In all the five galaxies the central regions display complex, distorted ionized gas velocity fields, though large scale ordered motions are present in three of them. With the current data we cannot determine whether these perturbed velocity fields are the signature of interaction/mergers episodes or they are the result of shocks, stellar winds or supernovas.

Further IFS observations are essential to investigate this issue: higher resolution spectra, in order to measure the velocity dispersion of the gas, as well as deeeper observations which allow to map the kinematics of the stars, are indeed fundamental.

- The galaxies studied here, although classified as BCDs, all show characteristics very different from those of "genuine" BCD galaxies: they have relatively high metallicities and significant amounts of dust; they show also variations of such properties 
as electron densities, extinction and ionization degrees, across the galaxies. These properties make them an especially attractive area, as they could be the local counterparts of the blue, high metallicity, vigorously starbursting galaxies, detected at intermediate redshift.

This paper is based on observations with the WHT, operated on the island of La Palma by the Royal Greenwich Observatory in the Spanish Observatorio del Roque de los Muchachos of the Instituto de Astrofísica de Canarias. We thank J. N. González-Pérez, J. M. Vílchez and P. Papaderos for their help in the initial stages of this project. We also thank J. AcostaPulido for assistance with the software we used to an- alyze the INTEGRAL data. We are grateful to the anonymous referee whose detailed review and criticism greatly helped to improve this paper. This research has made use of the NASA/IPAC Extragalactic Database (NED), which is operated by the Jet Propulsion Laboratory, Caltech, under contract with the National Aeronautics and Space Administration. This work has been partially funded by the Spanish "Ministerio de Ciencia y Tecnologia" (grants PB97-0158, AYA2001-3939, AYA2004-08260-C03-02, AyA2006-13682 and HA20060032). L. M. Cairós acknowledges support from the Alexander von Humboldt Foundation. N. Caon and C. Kehrig are grateful for the hospitality of the Astrophysikalisches Institut Potsdam, where part of this paper was written.

\section{APPENDIX}

\section{A. ATLAS OF SPECTRA}

Here for each galaxy we present a map with the spectrum, within a given wavelength interval, of each fiber, placed on the corresponding spatial position. The spectra have been rescaled to better show the line profile. 


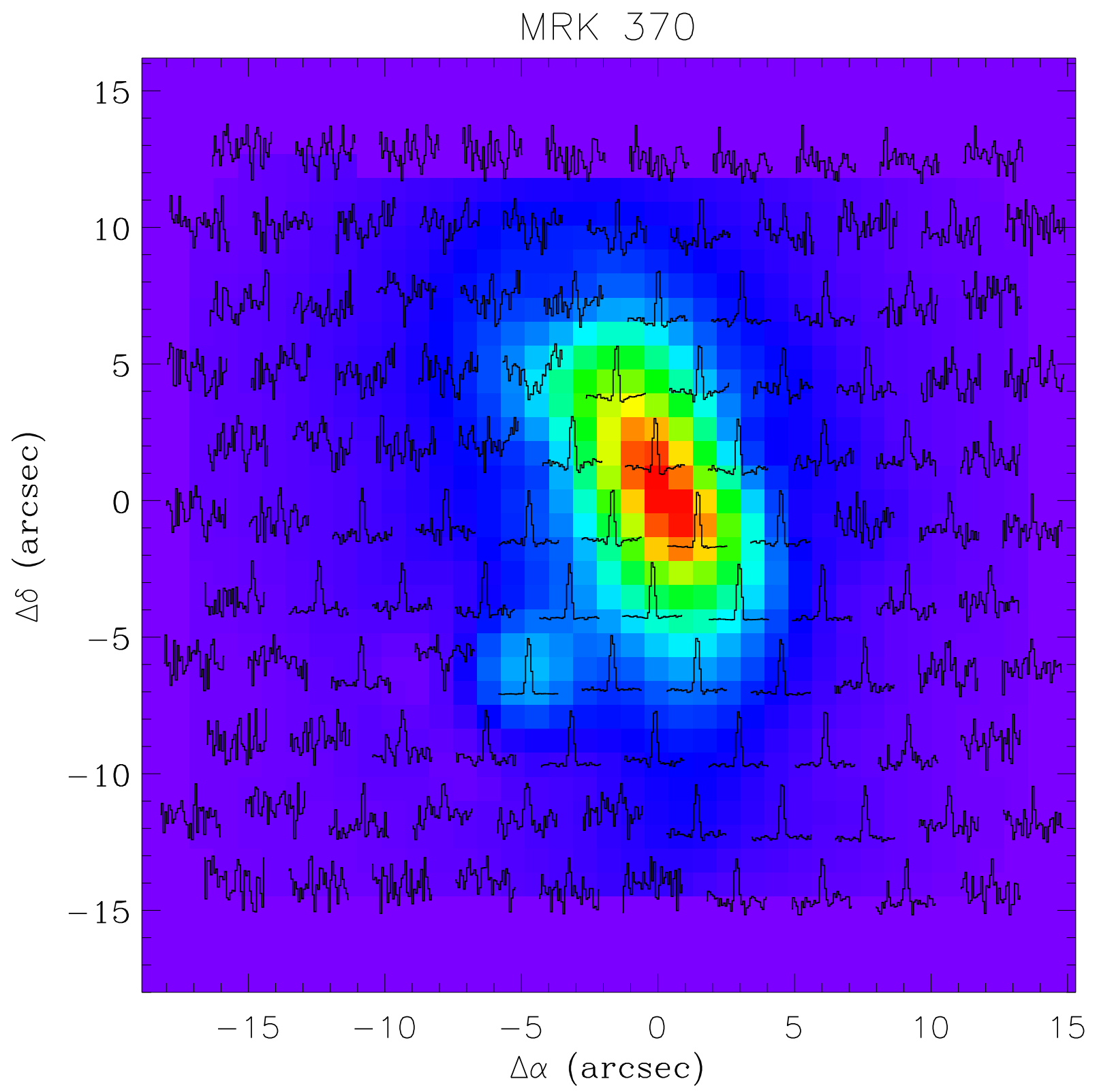

FIg. 10.- Two-dimensional spectrum diagrams for Mrk 370 in the spectral range 4820-4925 $\AA$, which includes H $\beta$. The spectra are over-plotted on the recovered V filter image, obtained by integrating the spectrum of each fiber within the wavelength interval of the Johnson-Cousin $V$-band filter. 


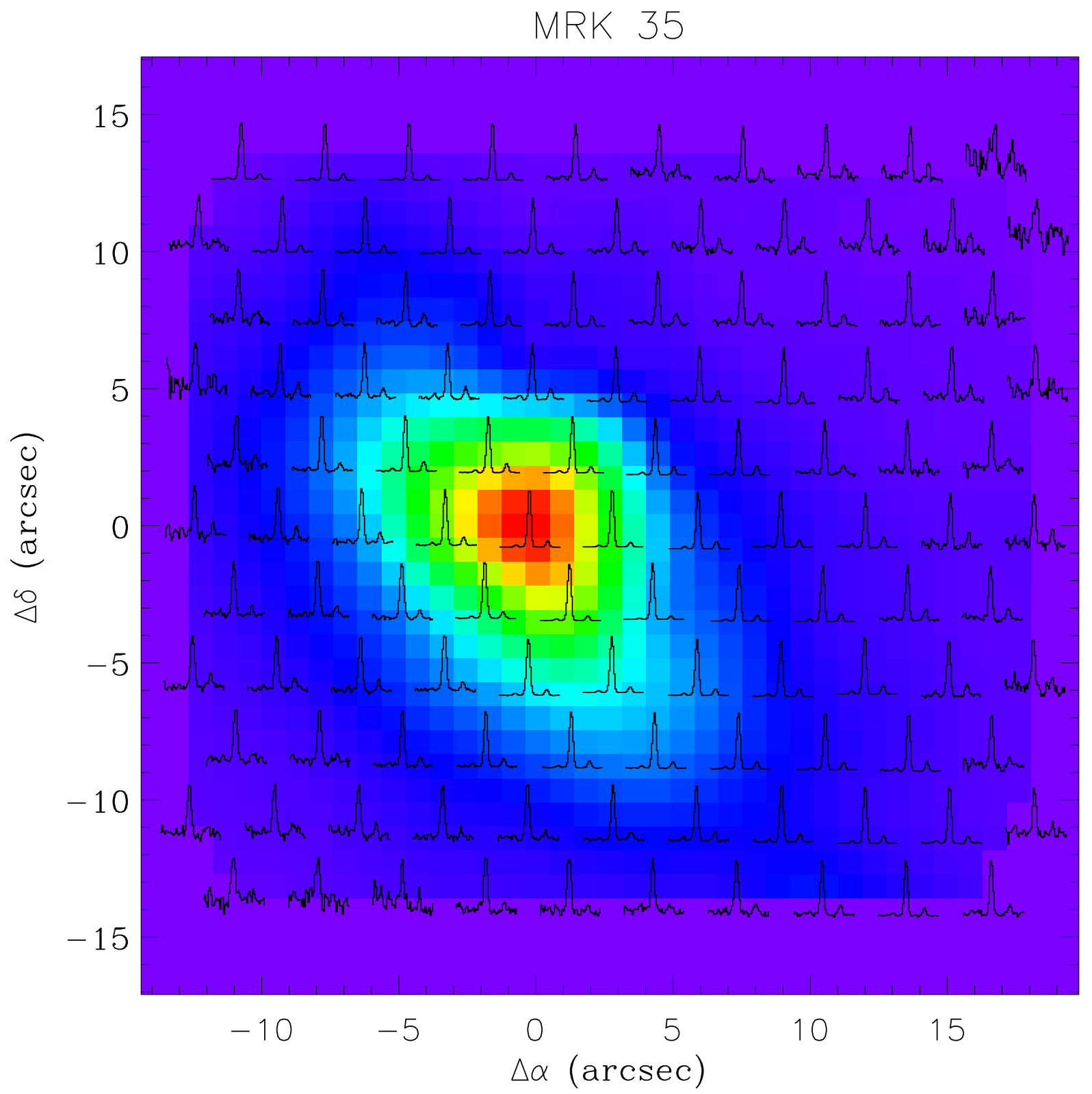

FIG. 11.- Two-dimensional spectrum diagrams for Mrk 35 in the spectral range 6540-6630 ̊, which includes H $\alpha+[\mathrm{N} \mathrm{II]} \lambda \lambda 6548,6584$. The spectra are over-plotted on the recovered $\mathrm{V}$ filter image. 


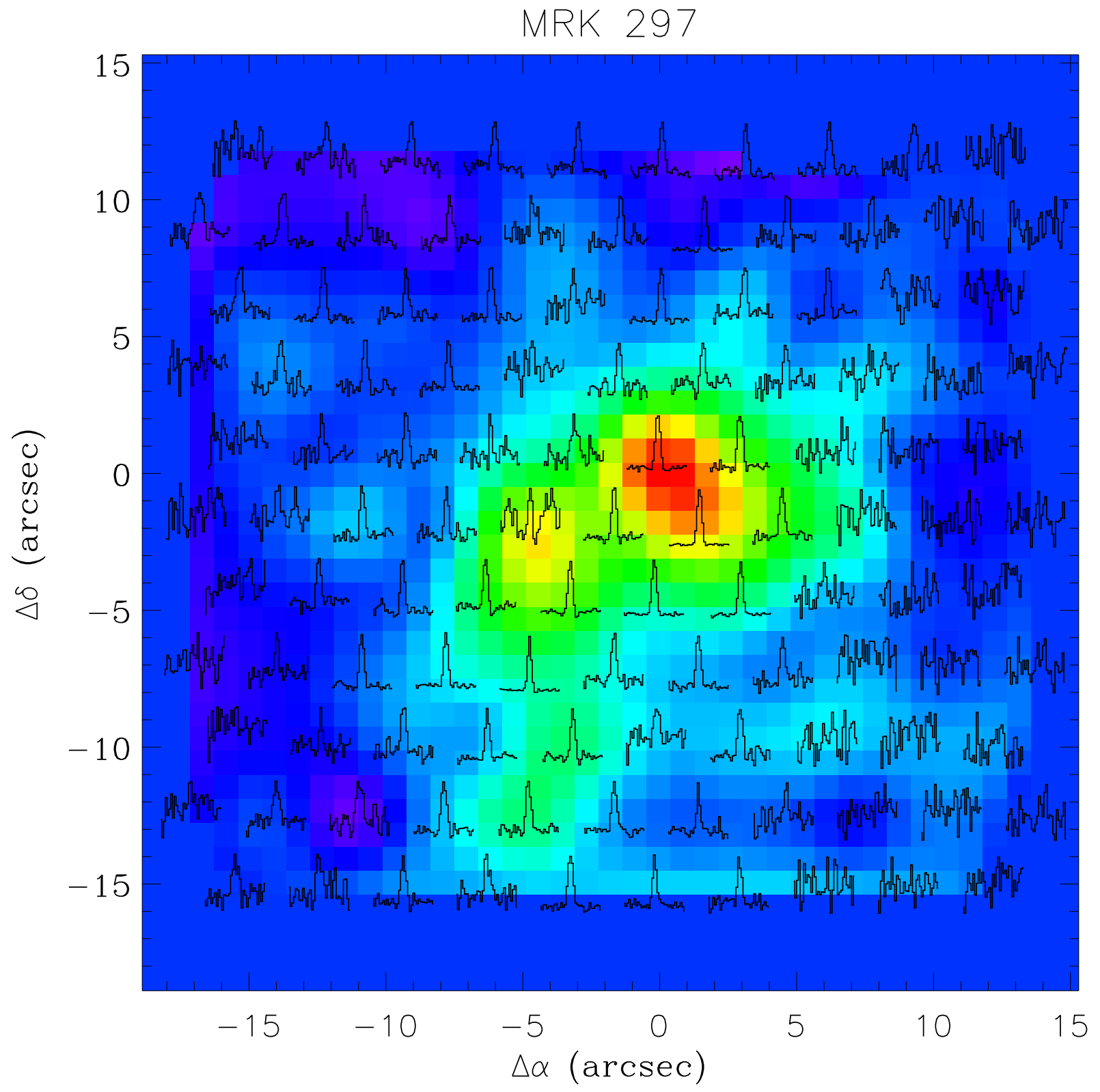

FIg. 12.- Two-dimensional spectrum diagrams for Mrk 297 in the spectral range 4885-4990 $\AA$, which includes H $\beta$. The spectra are over-plotted on the recovered V filter image. 


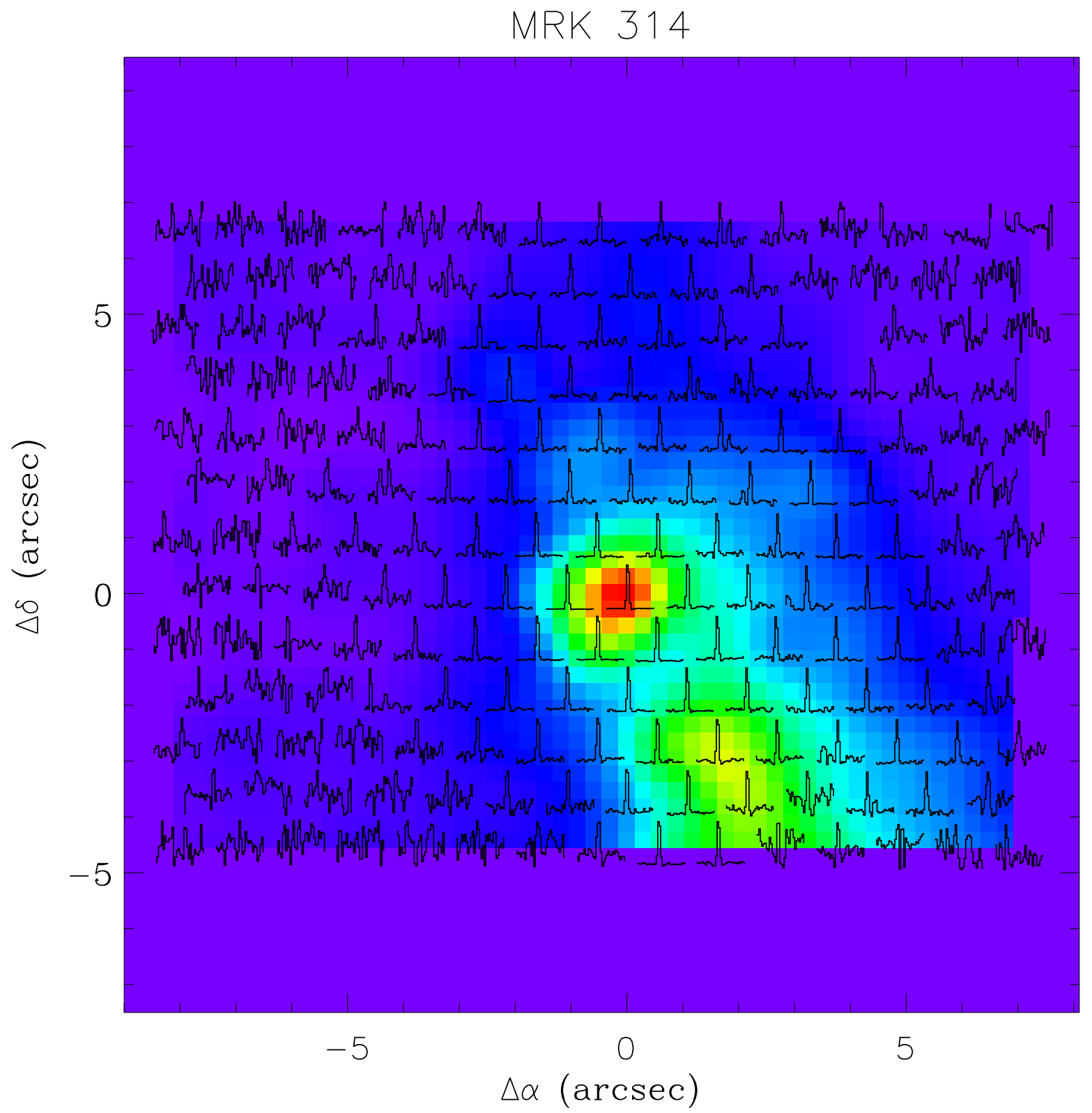

FIg. 13.- Two-dimensional spectrum diagrams for Mrk 314 in the spectral range 4850-4950 $\AA$, which includes H $\beta$. The spectra are over-plotted on the recovered V filter image. 


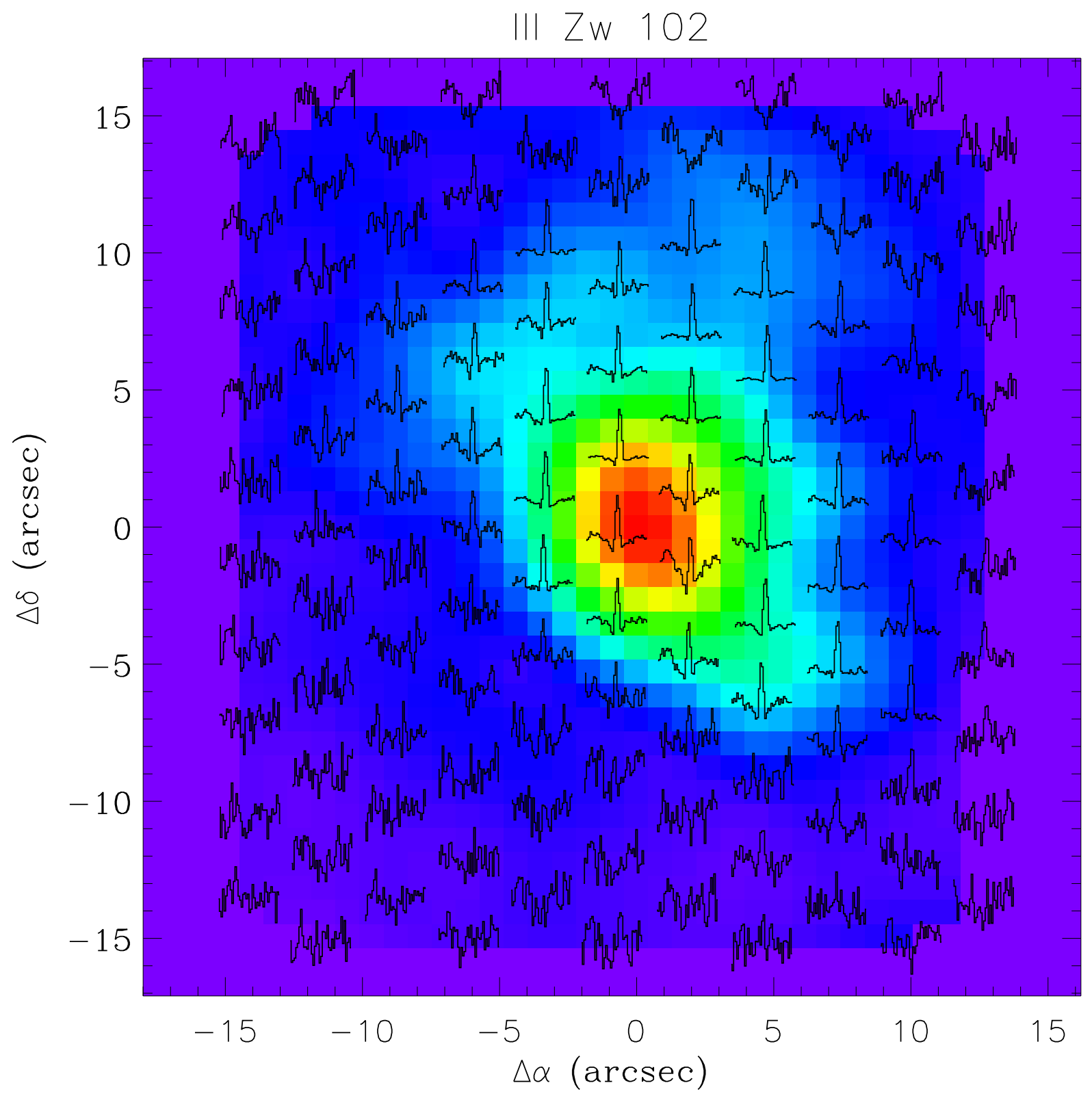

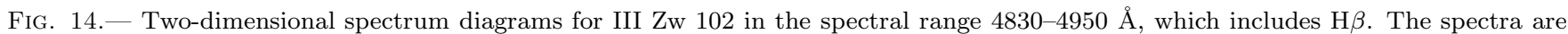
over-plotted on the recovered V filter image. 


\section{REFERENCES}

Allen, C. W. 1973, London: University of London, Athlone Press, 1973, 3rd ed.

Alloin, D., \& Duflot, R. 1979, A\&A, 78, L5

Arp, H. 1966, ApJS, 14, 1

Arribas, S., Mediavilla, E., Garcia-Lorenzo, B., \& del Burgo, C. 1997, ApJ, 490, 227

Arribas, S., Carter, D., Cavaller, L., del Burgo, C., Edwards, R., Fuentes, F. J., García, A. A., Herreros, J. M., Jones, L. R. Mediavilla, E., Pi, M., Pollacco, D., Rasilla, J. L., Rees, P. C., Sosa, N. A., et al. 1998, Proc. SPIE, 3355, 821

Arribas, S., Mediavilla, E., García-Lorenzo, B., del Burgo, C., \& Fuensalida, J. J. 1999, A\&AS, 136, 189

Baldwin, J. A., Phillips, M. M., \& Terlevich, R. 1981, PASP, 93, 5

Bergvall, N. \& Östlin, G. 2002, A\&A, 390, 891

Bingham, R. G., Gellatly, D. W., Jenkins, C. R., \& Worswick, S. P. 1994, Proc. SPIE, 2198, 56

Bravo-Alfaro, H., Brinks, E., Baker, A. J., Walter, F., \& Kunth, D. 2004, AJ, 127, 264

Bravo-Alfaro, H., Coziol, R., \& Brinks, E. 2006, Revista Mexicana de Astronomía y Astrofísica, 42, 261

Brosch, N., \& Loinger, F. 1991, A\&A, 249, 327

Burstein, D., \& Heiles, C. 1984, ApJS, 54, 33

Cairós, L. M. 2000, PhD Thesis, Instituto de Astrofísica de Canarias

Cairós, L. M., Vílchez, J. M., Gónzalez-Pérez, J. N., IglesiasPáramo, J., \& Caon, N. 2001a, ApJS, 133, 321(C01a)

Cairós, L. M., Caon, N., Vílchez, J. M., Gónzalez-Pérez, J. N., \& Muñoz-Tuñón, C. 2001b, ApJS, 136, 2(C01b)

Cairós, L. M., Caon, N., García-Lorenzo, B. , Vílchez, J. , \& MuñozTuñón, C. 2002, ApJ, 577, 164 (C02)

Cairós, L. M., Caon, N., Papaderos, P. Noeske, K. García-Lorenzo B. , Vílchez, J. , \& Muñoz-Tuñón, C. 2003, ApJ, 593, 312 (C03)

Cairós, L. M., Caon, N., García-Lorenzo, B., Monreal-Ibero, A., Amorín, R., Weilbacher, P., Papaderos, P. 2007, ApJ, 669, 251 (C07)

Calzetti, D., Kinney, A. L., \& Storchi-Bergmann, T. 1994, ApJ, 429,582

Campos-Aguilar, A., Moles, M., \& Masegosa, J. 1993, AJ, 106 1784

Caon, N., Cairós, L. M., Aguerri, J. A. L., \& Muñoz-Tuñón, C. 2005, ApJS, 157, 218

Copetti, M. V. F., Mallmann, J. A. H., Schmidt, A. A., \& Castañeda, H. O. 2000, A\&A, 357, 621

Davies, J.I., \& Phillipps, S. 1988, MNRAS, 233, 553

Deeg, H.-J., Duric, N., \& Brinks, E. 1997, A\&A, 323, 323

Demoulin, M.-H. 1969, ApJ, 157, 69

Denicoló, G., Terlevich, R., \& Terlevich, E. 2002, MNRAS, 330, 69

Fanelli, M. N., O'Connell, R. W., \& Thuan, T. X. 1988, ApJ, 334, 665

García-Lorenzo, B., Acosta-Pulido, J. A., \& Megías-Fernández, E. 2002, in ASP Conf. Ser. 282, Galaxies: The Third Dimension, ed. M. Rosado, L. Binette, \& L. Arias (San Francisco: ASP), 501

García-Lorenzo, B., Mediavilla, E., \& Arribas, S. 1999, ApJ, 518, 190

García-Lorenzo, B., Sánchez, S. F., Mediavilla, E., GonzálezSerrano, J. I., \& Christensen, L. 2005, ApJ, 621, 146

Garland, C. A., Pisano, D. J., Williams, J. P., Guzmán, R., \& Castander, F. J. 2004, ApJ, 615, 689

Gerola, H., Seiden, P. E., \& Schulman, L. S. 1980, BAAS, 12, 528 Gil de Paz, A., Zamorano, J., Gallego, J., \& Domínguez, F. 2000 A\&AS, 145,377

Gil de Paz, A., Madore, B. F., \& Pevunova, O. 2003, ApJS, 147, 29

Guseva, N. G., Papaderos, P., Izotov, Y. I., Green, R. F., Fricke, K. J., Thuan, T. X., \& Noeske, K. G. 2003a, A\&A, 407, 105

Guseva, N. G., Papaderos, P., Izotov, Y. I., Green, R. F., Fricke, K. J., Thuan, T. X., \& Noeske, K. G. 2003b, A\&A, 407, 91

Guseva, N. G., Papaderos, P., Izotov, Y. I., Green, R. F., Fricke, K. J., Thuan, T. X., \& Noeske, K. G. 2003c, A\&A, 407, 75

Johnson, K. E., Indebetouw, R., Watson, C., \& Kobulnicky, H. A. 2004, AJ, 128, 610

Kehrig, C., Vílchez, J. M., Sánchez, S. F., Telles, E., PérezMontero,E. \& Martín-Gordon, D. 2007, A\&A, accepted for publication
Kewley, L. J., Dopita, M. A., Sutherland, R. S., Heisler, C. A., \& Trevena, J. 2001, ApJ, 556, 121

Kunth, D., \& Sargent, W. L. W. 1983, ApJ, 273, 81

Kunth, D., Maurogordato, S., \& Vigroux, L. 1988, A\&A, 204, 10

Kunth, D., \& Östlin, G. 2000, A\&A Rev., 10, 1

Lequeux, J., Peimbert, M., Rayo, J. F., Serrano, A., \& TorresPeimbert, S. 1979, A\&A, 80, 155

Lodders, K. 2003, ApJ, 591, 1220

Loose, H. H. \& Thuan, T. X. 1986, in Star Forming Dwarf Galaxies and Related Objects, eds. D. Kunth, T. X. Thuan, J. T. T. Van (Gif-sur-Yvette: Editions Frontires), 73

Márquez, I., Boisson, C., Durret, F., \& Petitjean, P. 1998, A\&A, 333,459

Martínez-Delgado, I., Muñoz-Tuñón, C., Cairós, L. M. \& TenorioTagle, G. 2007, ApJ, submitted

Masegosa, J., Moles, M., \& Campos-Aguilar, A. 1994, ApJ, 420, 576

Mazzarella, J. M. \& Boroson, T. A. 1993, ApJS, 85, 27

McCall, M. L., Rybski, P. M., \& Shields, G. A. 1985, ApJS, 57, 1

Metcalfe, L., et al. 2005, A\&A, 444, 777

Moustakas, J., \& Kennicutt, R. C., Jr. 2006, ApJS, 164, 81

Mulchaey, J. S., Tsvetanov, Z., Wilson, A. S., \& Perez-Fournon, I. 1992, ApJ, 394, 91

Noeske, K. G., Iglesias-Páramo, J., Vílchez, J. M., Papaderos, P., \& Fricke, K. J. 2001, A\&A, 371, 806

Noeske, K. G., Papaderos, P., Cairós, L. M., \& Fricke, K. J. 2005, A\&A, 429, 115

Osterbrock, D. E., \& Ferland, G. J. 2006, Astrophysics of gaseous nebulae and active galactic nuclei, 2nd. ed. by D. E. Osterbrock and G. J. Ferland. Sausalito, CA: University Science Books, 2006

Östlin, G. 1998, Ph.D. Thesis

Östlin, G., Amram, P., Bergvall, N., Masegosa, J., Boulesteix, J., \& Márquez, I. 2001, A\&A, 374, 800

Papaderos, P., Loose, H.-H., Thuan, T. X., \& Fricke, K. J. 1996a, A\&AS, 120, 207

Papaderos, P., Ph.D. Thesis, Göttingen University

Papaderos, P., Fricke, K. J., Thuan, T. X., Izotov, Y. I., \& Nicklas, H. 1999, A\&A, 352, L57

Pilyugin, L. S. 2001, A\&A, 369, 594

Pustilnik, S. A., Kniazev, A. Y., Lipovetsky, V. A., \& Ugryumov, A. V. 2001, A'\&A, 373, 24

Sage, L. J., Loose, H. H., \& Salzer, J. J. 1993, A\&A, 273, 6

Schlegel, D. J., Finkbeiner, D. P.. \& Davis, M. 1998, ApJ, 500, 525

Searle, L. \& Sargent, W. L. W. 1972, ApJ, 173, 25

Shalyapina, L. V., Moiseev, A. V., Yakovleva, V. A., Hagen-Thorn, V. A., \& Barsunova, O. Y. 2004, Astronomy Letters, 30, 583

Shaw, R. A., \& Dufour, R. J. 1995, PASP, 107, 896

Shi, F., Kong, X., Li, C.. \& Cheng, F. Z. 2005, A\&A, 437, 849

Steel, S. J., Smith, N., Metcalfe, L., Rabbette, M., \& McBreen, B. 1996, A\&A, 311, 721

Storchi-Bergmann, T., Calzetti, D., \& Kinney, A. L. 1994, ApJ, 429, 572

Taniguchi, Y., \& Noguchi, M. 1991, AJ, 101, 1601

Taylor, C., Brinks, E., \& Skillman, E. D. 1993, AJ, 105, 128

Taylor, C. L., Brinks, E., Grashuis, R. M., \& Skillman, E. D. 1995 ApJS, 99, 427

Taylor, C. L., Thomas, D. L., Brinks, E., \& Skillman, E. D. 1996 , ApJS, 107, 143

Telles, E., \& Terlevich, R. 1995, MNRAS, 275, 1

Telles, E., \& Maddox, S. 2000, MNRAS, 311, 307

Thuan, T. X., \& Martin, G. E. 1981, ApJ, 247, 823

van Driel, W., Arnaboldi, M., Combes, F., \& Sparke, L. S. 2000, A\&AS, 141,385

Veilleux, S., \& Osterbrock, D. E. 1987, ApJS, 63, 295

Whelan, D. G., Devost, D., Charmandaris, V., Marshall, J. A., \& Houck, J. R. 2007, ApJ, 666, 896

Whitmore, B. C., Lucas, R. A., McElroy, D. B., Steiman-Cameron, T. Y., Sackett, P. D., \& Olling, R. P. 1990, AJ, 100, 1489 
TABLE 1

THE GALAXY SAMPLE

\begin{tabular}{|c|c|c|c|c|c|c|c|}
\hline $\begin{array}{c}\text { Galaxy } \\
(1)\end{array}$ & $\begin{array}{c}\text { Other names } \\
\text { (2) }\end{array}$ & $\begin{array}{l}\text { R.A. } \\
\text { (J2000) } \\
(3)\end{array}$ & $\begin{array}{c}\text { Decl. } \\
\text { (J2000) } \\
(4)\end{array}$ & $\begin{array}{c}D \\
(\mathrm{Mpc}) \\
(5)\end{array}$ & $\begin{array}{c}M_{B} \\
(\mathrm{mag}) \\
(6)\end{array}$ & $\begin{array}{c}\text { Morph. } \\
\text { (7) }\end{array}$ & $\begin{array}{c}A_{B} \\
(\mathrm{mag}) \\
(8)\end{array}$ \\
\hline Mrk 370 & NGC 1036, IC 1828,UGC 02160 & 024029.0 & +191750 & 10.8 & -17.00 & $\mathrm{Ch}$ & 0.399 \\
\hline Mrk 35 & NGC 3353 , UGC 05860 , Haro 3 , SBS $1042+562$ & 104522.4 & +555737 & 15.6 & -17.75 & $\mathrm{Ch}$ & 0.031 \\
\hline Mrk 297 & NGC 6052/6064, UGC 10182, Arp 209 & 160512.9 & +203232 & 65.1 & -21.05 & Ext & 0.330 \\
\hline Mrk 314 & NGC 7468, UGC 12329 & 230259.2 & +163619 & 28.9 & -18.53 & $\mathrm{Ch}$ & 0.368 \\
\hline III Zw 102 & NGC 7625, UGC 12529, Arp 212 & 232030.1 & +171332 & 22.7 & -19.28 & Ext & 0.109 \\
\hline
\end{tabular}

Note. - Columns (3) and (4): units of right ascension are hours, minutes, and seconds, and units of declination are degrees, arcminutes, and arcseconds. Col. (5): distance computed assuming a Hubble flow, with a Hubble constant $H_{0}=75 \mathrm{~km} \mathrm{sec}^{-1}$

$\mathrm{Mpc}^{-1}$, and applying the correction for the Local Group infall into Virgo. Col. (6): asymptotic photometry obtained by $\mathrm{Mpc}$, and applying the correction for the Local Group infall into Virgo. Col. (6): asymptotic photometry obtained by
extrapolating the growth curves, and corrected for galactic extinction (C01b). Col. (7): morphological classification following C01b. Col. (8): galactic extinction, from Schlegel, Finkbeiner, \& Davis (1998). 
TABLE 2

LOG OF THE OBSERVATIONS

\begin{tabular}{|c|c|c|c|c|c|c|c|c|}
\hline $\begin{array}{c}\text { Galaxy } \\
(1)\end{array}$ & $\begin{array}{c}\text { Date } \\
(2)\end{array}$ & $\begin{array}{c}\text { Bundle } \\
(3)\end{array}$ & $\begin{array}{c}\text { Spectral Range } \\
(\AA) \\
(4)\end{array}$ & $\begin{array}{c}\text { Fiber diameter } \\
(\operatorname{arcsec}) \\
(5)\end{array}$ & $\begin{array}{c}\text { Dispersion } \\
\left(\AA \text { pixel }^{-1}\right) \\
(6)\end{array}$ & $\begin{array}{l}\operatorname{texp} \\
(\mathrm{sec}) \\
(7)\end{array}$ & $\begin{array}{c}\text { Air mass } \\
(8)\end{array}$ & $\begin{array}{c}\text { Spatial scale } \\
\left(\mathrm{pc} /{ }^{\prime \prime}\right) \\
(9)\end{array}$ \\
\hline Mrk 370 & 1999 Aug 22 & STD3 & 4324-7304 & 2.7 & 2.99 & $3 \times 1200$ & $1.01-1.04$ & 53 \\
\hline Mrk 35 & 2003 Feb 25 & STD3 & $5481-6937$ & 2.7 & 1.43 & $3 \times 900$ & $1.16-1.19$ & 76 \\
\hline Mrk 297 & 1999 Aug 22 & STD3 & 4324-7304 & 2.7 & 2.99 & $2 \times 1200$ & $1.10-1.48$ & 313 \\
\hline Mrk 314 & 1999 Aug 22 & STD2 & $4414-7452$ & 0.9 & 2.99 & $3 \times 1800$ & $1.02-1.04$ & 140 \\
\hline III Zw 102 & 1999 Aug 22 & STD3 & $4324-7304$ & 2.7 & 2.99 & $3 \times 1800$ & $1.07-1.21$ & 110 \\
\hline
\end{tabular}


TABLE 3

The COMPUTED EMISSION LINE RATIOS IN THE DIFFERENT REGIONS

\begin{tabular}{|c|c|c|c|c|c|c|c|}
\hline Galaxy & Region & $\frac{\mathrm{H} \alpha}{\mathrm{H} \beta}$ & $\log \left(\frac{[\mathrm{O} \text { III }] \lambda 5007}{\mathrm{H} \beta}\right)$ & $\log \left(\frac{[\mathrm{N} \mathrm{II}] \lambda 6584}{\mathrm{H} \alpha}\right)$ & $\log \left(\frac{[\mathrm{S} \mathrm{II}] \lambda \lambda 6717,6731}{\mathrm{H} \alpha}\right)$ & $\log \left(\frac{[\mathrm{O} \mathrm{I}] \lambda 6300}{\mathrm{H} \alpha}\right)$ & $\frac{[\mathrm{S} \mathrm{II}] \lambda 6717}{[\mathrm{~S} \mathrm{II}] \lambda 6731}$ \\
\hline \multirow{4}{*}{ Mrk 370} & $\mathrm{~N}$ & $2.87 \pm 0.35$ & $0.26 \pm 0.02$ & $-0.84 \pm 0.02$ & $-0.65 \pm 0.02$ & $-2.06 \pm 0.07$ & $1.39 \pm 0.06$ \\
\hline & $\mathrm{R} 1$ & $2.66 \pm 0.33$ & $0.32 \pm 0.02$ & $-0.96 \pm 0.03$ & $-0.79 \pm 0.02$ & $\ldots$ & $1.33 \pm 0.04$ \\
\hline & $\mathrm{R} 2$ & $2.86 \pm 0.36$ & $0.30 \pm 0.02$ & $-0.88 \pm 0.03$ & $-0.68 \pm 0.02$ & $-2.07 \pm 0.09$ & $1.39 \pm 0.05$ \\
\hline & R3 & $2.93 \pm 0.38$ & $0.25 \pm 0.02$ & $-0.81 \pm 0.02$ & $-0.51 \pm 0.02$ & $\ldots$ & $1.41 \pm 0.05$ \\
\hline \multirow{4}{*}{ Mrk 35} & $\mathrm{~N}$ & $\cdots$ & $\cdots$ & $-1.22 \pm 0.02$ & $-1.02 \pm 0.02$ & $-2.13 \pm 0.07$ & $1.23 \pm 0.03$ \\
\hline & R1 & $\cdots$ & ... & $-1.09 \pm 0.02$ & $-0.87 \pm 0.02$ & $-1.97 \pm 0.05$ & $1.29 \pm 0.03$ \\
\hline & R2 & $\ldots$ & $\ldots$ & $-0.87 \pm 0.02$ & $-0.49 \pm 0.02$ & $-1.46 \pm 0.03$ & $1.39 \pm 0.04$ \\
\hline & R3 & $\ldots$ & $\ldots$ & $-1.00 \pm 0.01$ & $-0.72 \pm 0.02$ & $-1.78 \pm 0.04$ & $1.39 \pm 0.03$ \\
\hline \multirow{8}{*}{ Mrk 297} & $\mathrm{~N}$ & $3.91 \pm 1.50$ & $-0.11 \pm 0.19$ & $-0.67 \pm 0.03$ & $-0.40 \pm 0.03$ & $-1.39 \pm 0.11$ & $1.20 \pm 0.17$ \\
\hline & R1 & $2.59 \pm 0.37$ & $0.27 \pm 0.03$ & $-0.91 \pm 0.03$ & $-0.67 \pm 0.02$ & $-1.67 \pm 0.09$ & $1.43 \pm 0.07$ \\
\hline & $\mathrm{R} 2$ & $3.52 \pm 0.44$ & $0.31 \pm 0.02$ & $-0.87 \pm 0.01$ & $-0.65 \pm 0.02$ & $-1.50 \pm 0.04$ & $1.30 \pm 0.04$ \\
\hline & R3 & $2.99 \pm 0.39$ & $-0.22 \pm 0.03$ & $-0.66 \pm 0.01$ & $-0.54 \pm 0.02$ & $-1.43 \pm 0.05$ & $1.30 \pm 0.07$ \\
\hline & $\mathrm{R} 4$ & $5.12 \pm 0.77$ & $0.36 \pm 0.04$ & $-0.74 \pm 0.03$ & $-0.69 \pm 0.02$ & $-1.57 \pm 0.06$ & $1.23 \pm 0.10$ \\
\hline & R5 & $3.06 \pm 0.62$ & $0.22 \pm 0.07$ & $-0.86 \pm 0.03$ & $-0.61 \pm 0.04$ & $-1.38 \pm 0.07$ & $1.12 \pm 0.21$ \\
\hline & R6 & $3.38 \pm 0.47$ & $0.07 \pm 0.03$ & $-0.75 \pm 0.02$ & $-0.58 \pm 0.02$ & $-1.42 \pm 0.05$ & $1.26 \pm 0.07$ \\
\hline & R7 & $3.46 \pm 0.57$ & $0.26 \pm 0.05$ & $-0.88 \pm 0.02$ & $-0.66 \pm 0.02$ & $\ldots$ & $1.32 \pm 0.10$ \\
\hline \multirow[t]{6}{*}{ Mrk 314} & $\mathrm{~N}$ & $3.25 \pm 0.39$ & $0.65 \pm 0.01$ & $-1.34 \pm 0.03$ & $-0.94 \pm 0.02$ & $-2.02 \pm 0.05$ & $1.27 \pm 0.07$ \\
\hline & $\mathrm{R} 1$ & $3.11 \pm 0.37$ & $0.61 \pm 0.01$ & $-1.29 \pm 0.03$ & $-0.85 \pm 0.02$ & $-1.89 \pm 0.05$ & $1.33 \pm 0.05$ \\
\hline & $\mathrm{R} 2$ & $3.29 \pm 0.40$ & $0.54 \pm 0.02$ & $-1.23 \pm 0.02$ & $-0.74 \pm 0.02$ & $-1.80 \pm 0.05$ & $1.44 \pm 0.05$ \\
\hline & R3 & $2.90 \pm 0.38$ & $0.60 \pm 0.03$ & $-1.34 \pm 0.04$ & $-0.84 \pm 0.02$ & $\ldots$ & $1.31 \pm 0.10$ \\
\hline & R4 & $3.16 \pm 0.39$ & $0.46 \pm 0.02$ & $-1.12 \pm 0.03$ & $-0.69 \pm 0.02$ & $-1.63 \pm 0.08$ & $1.39 \pm 0.09$ \\
\hline & R5 & $3.03 \pm 0.38$ & $0.47 \pm 0.02$ & $-1.06 \pm 0.03$ & $-0.62 \pm 0.02$ & $-1.67 \pm 0.07$ & $1.25 \pm 0.09$ \\
\hline \multirow[t]{5}{*}{ III Zw 102} & $\mathrm{~N}$ & $6.30 \pm 0.82$ & $-0.78 \pm 0.11$ & $-0.37 \pm 0.01$ & $-0.59 \pm 0.02$ & & $1.23 \pm 0.06$ \\
\hline & $\mathrm{R} 1$ & $5.10 \pm 0.72$ & $-0.61 \pm 0.08$ & $-0.37 \pm 0.01$ & $-0.38 \pm 0.02$ & $-1.53 \pm 0.06$ & $1.13 \pm 0.08$ \\
\hline & $\mathrm{R} 2$ & $5.21 \pm 0.65$ & $-0.71 \pm 0.06$ & $-0.42 \pm 0.01$ & $-0.54 \pm 0.02$ & $\ldots$ & $1.29 \pm 0.06$ \\
\hline & R3 & $5.03 \pm 0.63$ & $-0.65 \pm 0.04$ & $-0.39 \pm 0.01$ & $-0.55 \pm 0.02$ & $-1.77 \pm 0.06$ & $1.34 \pm 0.05$ \\
\hline & $\mathrm{R} 4$ & $5.50 \pm 0.71$ & $-0.57 \pm 0.05$ & $-0.43 \pm 0.01$ & $-0.54 \pm 0.02$ & $\ldots$ & $1.36 \pm 0.06$ \\
\hline
\end{tabular}

Note. - The line ratios have been corrected for galactic extinction. The quoted uncertainties include both measurement errors and the uncertainty on the calibration factor, which is about $12 \%$ for the $\mathrm{H} \alpha / \mathrm{H} \beta$ ratio, $5 \%$ for [O III] $\lambda 5007 / \mathrm{H} \beta$, and $\leq 3 \%$ for the other ratios. 
TABLE 4

ELECTRON DENSITIES AND OXYGEN ABUNDANCES

\begin{tabular}{cccc}
\hline \hline Galaxy & Region & $N_{\mathrm{e}}$ & $12+\log (\mathrm{O} / \mathrm{H})$ \\
\hline Mrk 370 & $\mathrm{N}$ & $\leq 100$ & 8.51 \\
& $\mathrm{R} 1$ & $\leq 100$ & 8.42 \\
& $\mathrm{R} 2$ & $\leq 100$ & 8.48 \\
& $\mathrm{R} 3$ & $\leq 100$ & 8.53 \\
\hline Mrk 35 & $\mathrm{N}$ & 200 & 8.23 \\
& $\mathrm{R} 1$ & 131 & 8.32 \\
& $\mathrm{R} 2$ & $\leq 100$ & 8.48 \\
& $\mathrm{R} 3$ & $\leq 100$ & 8.40 \\
\hline Mrk 297 & $\mathrm{N}$ & 239 & 8.63 \\
& $\mathrm{R} 1$ & $\leq 100$ & 8.46 \\
& $\mathrm{R} 2$ & 120 & 8.48 \\
& $\mathrm{R} 3$ & 120 & 8.64 \\
& $\mathrm{R} 4$ & 200 & 8.58 \\
& $\mathrm{R} 5$ & 360 & 8.49 \\
& $\mathrm{R} 6$ & 165 & 8.57 \\
& $\mathrm{R} 7$ & 100 & 8.48 \\
\hline Mrk 314 & $\mathrm{N}$ & 153 & 8.14 \\
& $\mathrm{R} 1$ & $\leq 100$ & 8.18 \\
& $\mathrm{R} 2$ & $\ldots$ & 8.22 \\
& $\mathrm{R} 3$ & 110 & 8.14 \\
& $\mathrm{R} 4$ & $\leq 100$ & 8.30 \\
& $\mathrm{R} 5$ & 176 & 8.35 \\
\hline III Zw 102 & $\mathrm{N}$ & 200 & 8.85 \\
& $\mathrm{R} 1$ & 343 & 8.84 \\
& $\mathrm{R} 2$ & 130 & 8.81 \\
& $\mathrm{R} 3$ & $\leq 100$ & 8.83 \\
& $\mathrm{R} 4$ & $\leq 100$ & 8.81 \\
& & & \\
\hline
\end{tabular}

Note. - Abundances were computed using the empirical calibrations published in Denicoló et al. (2002), whose typical uncertainty is \pm 0.2 dex. 
TABLE 5

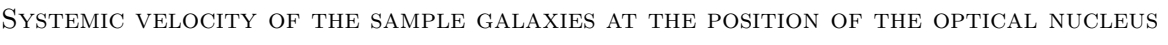

\begin{tabular}{|c|c|c|c|c|c|}
\hline $\begin{array}{c}\text { Galaxy } \\
(1)\end{array}$ & $\begin{array}{c}V_{\text {sys }}\left(\mathrm{km} \mathrm{s}^{-1}\right) \\
(2)\end{array}$ & 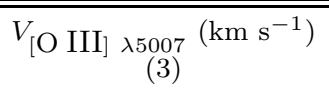 & 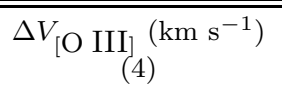 & $\begin{array}{c}V_{\mathrm{H} \alpha}\left(\mathrm{km} \mathrm{s}^{-1}\right) \\
(5)\end{array}$ & $\begin{array}{c}\Delta V_{\mathrm{H} \alpha}\left(\mathrm{km} \mathrm{s}^{-1}\right) \\
(6)\end{array}$ \\
\hline Mrk 370 & 790 & $715 \pm 6$ & $35 \pm 9$ & $\begin{array}{l}735 \pm 5 \\
958+5\end{array}$ & $\begin{array}{l}28 \pm 5 \\
31+7\end{array}$ \\
\hline $\begin{array}{l}\text { Mrk } 35 \\
\text { Mrk } 297\end{array}$ & $\begin{array}{r}944 \\
4739\end{array}$ & $4720 \pm 9$ & $125 \pm 14$ & $\begin{array}{r}958 \pm 5 \\
4714 \pm 5\end{array}$ & $\begin{array}{r}31 \pm 7 \\
118 \pm 7\end{array}$ \\
\hline Mrk 314 & 2081 & $2117 \pm 6$ & $58 \pm 8$ & $2104 \pm 5$ & $52 \pm 7$ \\
\hline III Zw 102 & 1633 & $1463 \pm 37$ & $254 \pm 50$ & $1588 \pm 5$ & $66 \pm 7$ \\
\hline
\end{tabular}

2) lists the systemic velocity from NASA Extragalac

(http://nedwww.ipac.caltech.edu/). Cols. (4) and (6) list the half velocity amplitude in the central 5 arcsec

of the galaxy. 


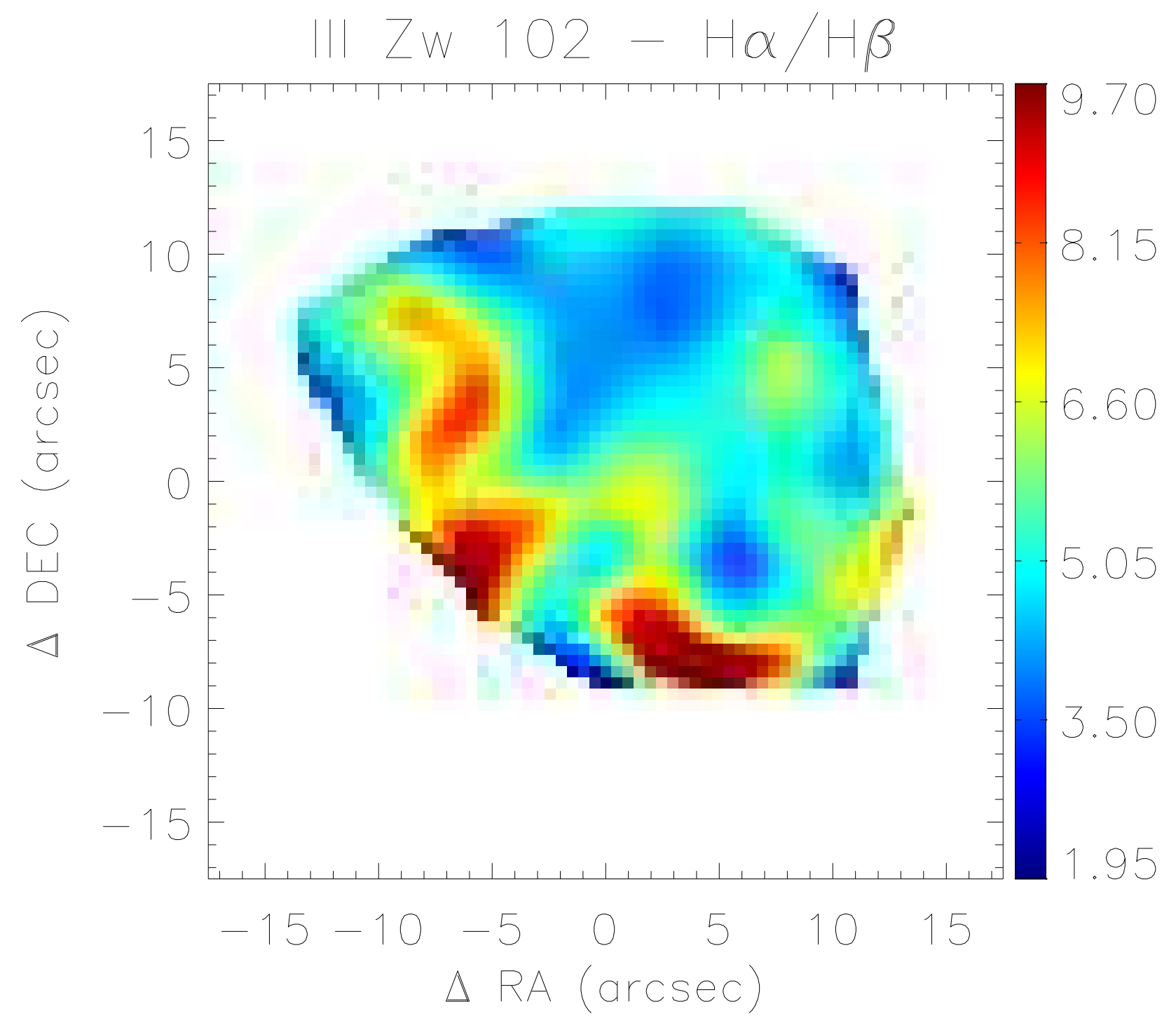

